\title{
Existence of positive solutions for discrete delta-nabla fractional boundary value problems with $p$-Laplacian
}

Huan Liu, Yuanfeng Jin and Chengmin Hou*

\section{"Correspondence:}

cmhou@foxmail.com Department of Mathematics,

Yanbian University, Yanji, 133002, P.R. China

\section{然 Springer}

$$
\begin{aligned}
& \text { Abstract } \\
& \text { In this paper, we consider a discrete delta-nabla boundary value problem for the } \\
& \text { fractional difference equation with } p \text {-Laplacian } \\
& \qquad \triangle_{v-2}^{\beta}\left(\varphi_{p}\left({ }_{b} \nabla^{v} x(t)\right)\right)+\lambda f\left(t-v+\beta+1, x(t-v+\beta+1),\left[{ }_{b} \nabla^{\varepsilon} x(t)\right]_{t-v+\beta+\varepsilon+1}\right)=0, \\
& \qquad x(b)=0, \quad\left[{ }_{b} \nabla^{v} x(t)\right]_{v-2}=0, \quad x(-1)=\sum_{t=0}^{b-1} x(t) A(t),
\end{aligned}
$$

where $t \in \mathbb{T}=[v-\beta-1, b+v-\beta-1]_{\mathbb{N}_{v-\beta-1}} . \Delta_{v-2}^{\beta}, b \nabla^{v}$ are left and right fractional difference operators, respectively, and $\varphi_{p}(s)=|s|^{p-2} s, p>1$.

By using the method of upper and lower solution and the Schauder fixed point theorem, we obtain the existence of positive solutions for the above boundary value problem; and applying a monotone iterative technique, we establish iterative schemes for approximating the solution.

MSC: 39A06; 39A22

Keywords: discrete delta-nabla; boundary value problem; positive solutions; upper and lower solution; monotone iteration; fixed point theorem

\section{Introduction}

In this paper, we investigate the existence of positive solutions for the following discrete delta-nabla fractional boundary value problem (FBVP) with $p$-Laplacian:

$$
\begin{aligned}
& \triangle_{v-2}^{\beta}\left(\varphi_{p}\left({ }_{b} \nabla^{v} x(t)\right)\right)+\lambda f\left(t-v+\beta+1, x(t-v+\beta+1),\left[{ }_{b} \nabla^{\varepsilon} x(t)\right]_{t-v+\beta+\varepsilon+1}\right) \\
& \quad=0, \quad t \in \mathbb{T}, \\
& x(b)=0, \quad\left[{ }_{b} \nabla^{v} x(t)\right]_{v-2}=0, \quad x(-1)=\sum_{t=0}^{b-1} x(t) A(t),
\end{aligned}
$$

where $t \in \mathbb{T}=[v-\beta-1, b+v-\beta-1]_{\mathbb{N}_{v-\beta-1}}, \triangle_{\nu-2}^{\beta},{ }_{b} \nabla^{v}$ are left and right fractional difference operators, respectively. $\varphi_{p}(s)=|s|^{p-2} s, p>1, \varphi_{p}^{-1}=\varphi_{q}, \frac{1}{p}+\frac{1}{q}=1, \beta, \nu, \varepsilon \in(0,+\infty)$, and they satisfy the following:

(c) The Author(s) 2017. This article is distributed under the terms of the Creative Commons Attribution 4.0 International License (http://creativecommons.org/licenses/by/4.0/), which permits unrestricted use, distribution, and reproduction in any medium, provided you give appropriate credit to the original author(s) and the source, provide a link to the Creative Commons license, and indicate if changes were made. 
$\left(H_{1}\right) \quad v \in(1,2], \beta, \varepsilon \in(0,1], v-\varepsilon-1>0$;

$\left(H_{2}\right) A(t)$ is a function defined on $[0, b]_{\mathbb{N}_{0}}$;

$\left(H_{3}\right) f:[0, b]_{\mathbb{N}_{0}} \times \mathbb{R} \times \mathbb{R} \longrightarrow[0, \infty)$ is continuous for any $t \in[0, b]_{\mathbb{N}_{0}}, f(t, 0,0) \neq 0$, $f(t, 1,1) \neq 0$, and let

$$
\sigma=\max _{t \in[0, b]_{\mathbb{N}_{0}}} f(t, 1,1) \neq 0
$$

The equation with $p$-Laplacian operator arises in the modeling of different physical and natural phenomena, non-Newtonian mechanics [1], combustion theory [2], population biology [3], nonlinear flow laws [4] and the system of Monge-Kantorovich mass transfer [5]. Integral and derivative operators of fractional order can describe the characteristics exhibited in many complex processes and systems having long-memory in time. Then many classical integer order models for complex systems are substituted by fractional order models. Fractional calculus has recently developed into a relatively vibrant research area. It also provides an excellent tool to describe the hereditary properties of materials and processes. Many successful new applications of fractional calculus in various fields have also been reported recently. For example, Nieto and Pimentel [6] extended a secondorder thermostat model to the fractional model; Ding and Jiang [7] used waveform relaxation methods to study some fractional functional differential equation models. For the basic theories of fractional calculus and some recent work in application, the reader is referred to Refs. [8-13].

On the other hand, discrete fractional calculus has attracted slowly but steadily increasing attention in the past seven years or so. In particular, several recent papers by Atici and Eloe as well as other recent papers by the present authors have addressed some basic theory of both discrete fractional initial value problems and discrete FBVPs. More specifically, Atici and Eloe [14] have already analyzed a transform method in discrete fractional calculus. Goodrich [15] considered a discrete right-focal fractional boundary value problem. All of the fundamental background in discrete fractional calculus can be found in [16] which is written by Goodrich and Peterson. Other recent work has considered discrete FBVPs with a variety of boundary conditions, see [17-20]. There are also a few papers for the discrete delta-nabla boundary value problems. For example, Malinowska and Torres [21] propose a more general approach to the calculus of variations on time scales that allows to obtain both delta and nabla results as particular cases. Martins and Torres [22] study the calculus of variations on time scales with nabla derivatives and so on. What is more, [23] is the first paper to consider a discrete fractional difference equation with a $p$-Laplacian operator.

From the above works, we can see the fact that although the discrete delta-nabla boundary value problem has been studied by many authors, to the best of our knowledge, there are very few papers on the discrete delta-nabla FBVPs. For example, Xie, Jin and Hou [24] obtained some results which ensure the existence of a well precise interval of the parameter for which the problem admits multiple solutions.

Our aim is to use the method of upper and lower solution and the Schauder fixed point theorem to obtain the existence of positive solutions for the above boundary value problem; and to apply a monotone iterative technique to establish iterative schemes for approximating the solution. 
The rest of this paper has the following structure. In Section 2, we recall some basic definitions of fractional calculus, establish some lemmas and use symbols to replace with some formula which plays a pivotal role in the text. Section 3 contains an existence result for problem (1.1) and (1.2) which is established by applying the method of upper and lower solution and the Schauder fixed point theorem. In Section 4, we show the iterative schemes for approximating the solution by using a monotone iterative technique.

\section{Preliminaries}

In this section, we collect some basic definitions and lemmas for manipulating discrete fractional operators.

For any real number $\beta$, let $\mathbb{N}_{\beta}=\{\beta, \beta+1, \beta+2, \ldots\},{ }_{\beta} \mathbb{N}=\{\ldots, \beta-2, \beta-1, \beta\}$.

We define $t^{\underline{v}}=\frac{\Gamma(t+1)}{\Gamma(t+1-v)}$ for any $t, v \in \mathbb{R}$, for which the right-hand side is well defined. We also appeal to the convention that if $t+1-v$ is a pole of the gamma function and $t+1$ is not a pole, then $t^{\underline{\underline{v}}}=0$.

Definition 2.1 ([17]) Let $f: \mathbb{N}_{a} \rightarrow \mathbb{R}$ and $v>0$ be given. The $v$ th left fractional sum of $f$ is given by

$$
\triangle_{a}^{-v} f(t)=\frac{1}{\Gamma(\nu)} \sum_{s=a}^{t-v}(t-s-1)^{\frac{\nu-1}{}} f(s) \quad \text { for } t \in \mathbb{N}_{a+\nu} .
$$

Also, let $N \in \mathbb{N}$ be chosen such that $N-1<v \leq N$. Then the $v$ th left fractional difference of $f$ is given by

$$
\triangle_{a}^{v} f(t):=\triangle^{N} \triangle_{a}^{v-N} f(t) \text { for } t \in \mathbb{N}_{a+N-v}
$$

Definition 2.2 ([18]) The $v$ th right fractional sum of $f(t)$ for $v>0$ is defined by

$$
{ }_{b} \nabla^{-v} f(t)=\frac{1}{\Gamma(v)} \sum_{s=t+v}^{b}(s-t-1)^{\frac{v-1}{f}} f(s) \quad \text { for } t \in_{b-v} \mathbb{N} .
$$

We also define the $v$ th right fractional difference for $v>0$ by

$$
{ }_{b} \nabla^{v} f(t):=(-1)^{N} \nabla_{b}^{N} \nabla^{v-N} f(t) \quad \text { for } t \in_{b-N+v} \mathbb{N},
$$

where $N \in \mathbb{N}$ is chosen so that $0 \leq N-1<v \leq N$.

Lemma 2.1 ([18]) Let $b \in \mathbb{R}$ and $\mu>0$ be given. Then

$$
\nabla(b-t)^{\underline{\mu}}=-\mu(b-t) \underline{\mu-1}
$$

for any $t$, for which both sides are well defined.

Furthermore, for $v>0$ with $N-1<v \leq N, N \in \mathbb{N}$,

$$
{ }_{b-\mu} \nabla^{-v}(b-t)^{\underline{\mu}}=\mu^{-\underline{v}}(b-t)^{\underline{\mu+v}}, \quad t \in \in_{b-\mu-v} \mathbb{N},
$$


and

$$
{ }_{b-\mu} \nabla^{v}(b-t)^{\mu}=\mu^{\underline{v}}(b-t) \stackrel{\mu-v}{\underline{n}}, \quad t \in{ }_{b-\mu-N+v} \mathbb{N} .
$$

Lemma 2.2 ([17]) Let $f: \mathbb{N}_{a} \rightarrow \mathbb{R}$ be given, and suppose $v, \mu>0$ with $N-1<v \leq N$.

Then

$$
\triangle_{a+\mu}^{v} \triangle_{a}^{-\mu} f(t)=\triangle_{a}^{\nu-\mu} f(t), \quad t \in \mathbb{N}_{a+\mu+N-v}
$$

Lemma 2.3 ([18]) Let $f:{ }_{b} \mathbb{N} \rightarrow \mathbb{R}$ be given, and suppose $v, \mu>0$ with $N-1<v \leq N$.

Then

$$
{ }_{b-\mu} \nabla_{b}^{v} \nabla^{-\mu} f(t)={ }_{b} \nabla^{\nu-\mu} f(t), \quad t \in{ }_{b-\mu-N+\nu} \mathbb{N} .
$$

Lemma 2.4 ([17]) Let $f: \mathbb{N}_{a} \rightarrow \mathbb{R}$ and $v>0$ be given with $N-1<v \leq N$. The following two definitions for the left fractional difference $\Delta_{a}^{v} f: \mathbb{N}_{a+N-v} \rightarrow \mathbb{R}$ are equivalent:

$$
\begin{aligned}
& \Delta_{a}^{v} f(t)=\Delta^{N} \Delta_{a}^{-(N-v)} f(t), \\
& \Delta_{a}^{v} f(t)= \begin{cases}\frac{1}{\Gamma(-v)} \sum_{s=a}^{t+v}(t-s-1) \frac{-v-1}{-} f(s), & N-1<v \leq N, \\
\Delta^{N} f(t), & v=N .\end{cases}
\end{aligned}
$$

Lemma 2.5 ([18]) Let $f:{ }_{b} \mathbb{N} \rightarrow \mathbb{R}$ and $v>0$ be given with $N-1<v \leq N$. The following two definitions for the right fractional difference ${ }_{b} \nabla^{v} f:{ }_{b-N+v} \mathbb{N} \rightarrow \mathbb{R}$ are equivalent:

$$
\begin{aligned}
& { }_{b} \nabla^{v} f(t)=(-1)^{N} \nabla_{b}^{N} \nabla^{-(N-v)} f(t), \\
& { }_{b} \nabla^{v} f(t)= \begin{cases}\frac{1}{\Gamma(-v)} \sum_{s=t-v}^{b}(s-t-1) \stackrel{-v-1}{b} f(s), & N-1<v \leq N, \\
(-1)^{N} \nabla^{N} f(t), & v=N .\end{cases}
\end{aligned}
$$

Lemma 2.6 ([17]) Let $f: \mathbb{N}_{a} \rightarrow \mathbb{R}$ be given and suppose $k \in \mathbb{N}_{0}$ and $v>0$. Then for $t \in$ $\mathbb{N}_{a+M-\mu+v}$,

$$
\triangle_{a}^{-v} \triangle^{k} f(t)=\triangle_{a}^{k-v} f(t)-\sum_{j=0}^{k-1} \frac{\triangle^{j} f(a)}{\Gamma(v-k+j+1)}(t-a) \frac{v-k+j}{} .
$$

Moreover, if $\mu>0$ with $M-1<\mu \leq M$, then for $t \in \mathbb{N}_{a+v}$,

$$
\triangle_{a+M-\mu}^{-v} \triangle_{a}^{\mu} f(t)=\triangle_{a}^{\mu-v} f(t)-\sum_{j=0}^{M-1} \frac{\triangle_{a}^{j-M+\mu} f(a+M-\mu)}{\Gamma(v-M+j+1)}(t-a-M+\mu) \frac{v-M+j}{.}
$$

Lemma $2.7([18])$ Let $:{ }_{b} \mathbb{N} \rightarrow \mathbb{R}$ be given, and suppose $k \in \mathbb{N}_{0}$ and $v>0$. Then for $t \in{ }_{b-v} \mathbb{N}$,

$$
{ }_{b} \nabla^{-v}{ }_{b} \nabla^{k} f(t)={ }_{b} \nabla^{k-v} f(t)-\sum_{j=0}^{k-1} \frac{{ }_{b} \nabla^{j} f(b)}{\Gamma(v-k+j+1)}(b-t) \stackrel{v-k+j}{ } .
$$


Moreover, if $\mu>0$ with $M-1<\mu \leq M$, then for $t \in b_{b-M+\mu-\nu} \mathbb{N}$,

$$
{ }_{b-M+\mu} \nabla^{-v}{ }_{b} \nabla^{\mu} f(t)={ }_{b} \nabla^{\mu-v} f(t)-\sum_{j=0}^{M-1} \frac{{ }_{b} \nabla^{j-M+\mu} f(b-M+\mu)}{\Gamma(v-M+j+1)}(b-M+\mu-t) \frac{\nu-M+j}{} .
$$

Remark 2.1 When we choose $t \in \mathbb{T}=[v-\beta-1, b+v-\beta-1]_{\mathbb{N}_{\nu-\beta-1}}$ in (1.1), problem (1.1) (1.2) is significative. In fact, by Definitions $2.1,2.2$, Lemmas 2.4 and 2.5 , we have

$$
\triangle_{\nu-2}^{\beta} \varphi_{p}\left({ }_{b} \nabla^{v} x(t)\right)=\frac{1}{\Gamma(-\beta)} \sum_{s=v-2}^{t+\beta}(t-s-1) \frac{-\beta-1}{-} \varphi_{p}\left(\frac{1}{\Gamma(-v)} \sum_{u=s-v}^{b}(u-s-1) \frac{-v-1}{x} x(u)\right),
$$

and

$$
\left[{ }_{b} \nabla^{\varepsilon} x(t)\right]_{t-v+\beta+\varepsilon+1}=\frac{1}{\Gamma(-\varepsilon)} \sum_{s=t-v+\beta+1}^{b}(s+v-\beta-\varepsilon-t-2)^{-\frac{\varepsilon-1}{x}} x(s) .
$$

We can see that the domain of the function $x$ is $\{-2,-1,0, \ldots, b\}$.

In the following paragraphs, we define $\sum_{t=i}^{j} y(t)=0$ for $j<i$.

Next, we denote

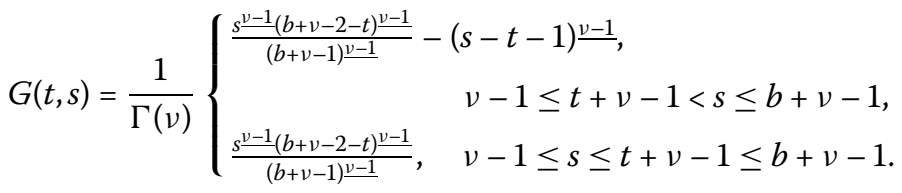

$$
\begin{aligned}
& G_{A}(s)=\sum_{t=0}^{b-1} \frac{G(t, s) A(t)}{(b+v-1) \underline{v-1}}, \quad C=\sum_{t=0}^{b-1} \frac{(b+v-2-t)^{\frac{v-1}{}}}{(b+v-1) \underline{v-1}} A(t) \\
& J(t, s)=\frac{(b+v-2-t)^{\frac{v-1}{2}}}{(1-C) \Gamma(v)} G_{A}(s)+G(t, s) .
\end{aligned}
$$

For variable $t$, we denote

$$
t^{\prime}=t-v+\beta+1, \quad t^{\prime \prime}=b+2 v-t-\beta-3
$$

By Lemmas 2.1 and 2.5, for $v-1 \leq s \leq b+v-1$, we have

$$
\begin{aligned}
& { }_{b} \nabla_{t}^{\varepsilon}(s-t-1)^{\frac{\nu-1}{}}=\frac{1}{\Gamma(-\varepsilon)} \sum_{u=t-\varepsilon}^{b}(u-t-1)^{\frac{-\varepsilon-1}{}}(s-u-1)^{\frac{\nu-1}{}} \\
& =\frac{1}{\Gamma(-\varepsilon)} \sum_{u=t-\varepsilon}^{s-v}(u-t-1)^{\frac{-\varepsilon-1}{2}}(s-u-1)^{\frac{\nu-1}{}} \\
& ={ }_{s-v} \nabla_{t}^{\varepsilon}(s-t-1)^{\frac{\nu-1}{}} \\
& =(\nu-1)^{\underline{\varepsilon}}(s-t-1)^{\underline{\nu-\varepsilon-1}} \\
& =\frac{\Gamma(\nu)}{\Gamma(v-\varepsilon)}(s-t-1)^{\frac{v-\varepsilon-1}{}} \text {. }
\end{aligned}
$$


Thus

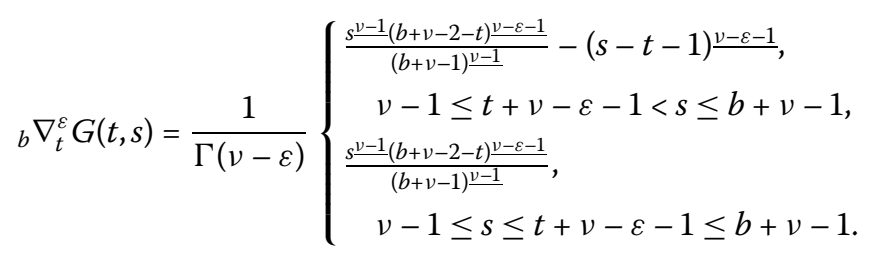

We denote

$$
\bar{G}(t, s):={ }_{b} \nabla_{t}^{\varepsilon} G(t, s), \quad \bar{J}(t, s):=\bar{G}(t, s)+\frac{(b+v-2-t)^{\frac{v-\varepsilon-1}{}}}{(1-C) \Gamma(v-\varepsilon)} G_{A}(s) .
$$

Lemma 2.8 Let $0 \leq C<1$ and $h:[0, b]_{\mathbb{N}_{0}} \rightarrow \mathbb{R}$ be given, the problem

$$
\left\{\begin{array}{l}
{ }_{b} \nabla^{v} x(t)+h(t-v+1)=0, \quad t \in[v-1, b+v-1]_{\mathbb{N}_{v-1}}, \\
x(b)=0, \quad x(-1)=\sum_{t=0}^{b-1} x(t) A(t)
\end{array}\right.
$$

has the unique solution

$$
x(t)=\sum_{s=v-1}^{b+v-2} J(t, s) h(s-v+1)
$$

where $J(t, s)$ is given by (2.2).

Proof Denote

$$
\bar{h}(s)=\frac{h(s-v+1)}{(b+v-1) \frac{v-1}{}} .
$$

By Lemma 2.7, we have

$$
x(t)={ }_{b+v-2} \nabla^{-v} h(t-v+1)+k_{1}(b+v-2-t)^{\frac{v-1}{2}}+k_{2}(b+v-2-t)^{\frac{v-2}{}} .
$$

From (2.5), we have $k_{2}=0$ and

$$
k_{1}=\frac{1}{(1-C) \Gamma(v)}\left(\sum_{s=v-1}^{b+v-2} s^{\frac{v-1}{1}} h(s)-\sum_{t=0}^{b-1} A(t) \sum_{s=t+v}^{b+v-2}(s-t-1)^{\frac{v-1}{h}}(s)\right)
$$

Then

$$
\begin{aligned}
& x(t)=-\frac{1}{\Gamma(v)} \sum_{s=t+v}^{b+v-2}(s-t-1)^{\frac{v-1}{w}} h(s-v+1) \\
& +\frac{(b+v-2-t)^{\frac{\nu-1}{2}}}{(1-C) \Gamma(v)}\left(\sum_{s=v-1}^{b+v-2} s^{\frac{\nu-1}{h}}(s)-\sum_{t=0}^{b-1} A(t) \sum_{s=t+v}^{b+v-2}(s-t-1)^{\frac{v-1}{h}}(s)\right) \\
& =\sum_{s=v-1}^{b+v-2} G(t, s) h(s-v+1)-\frac{1}{\Gamma(v)} \sum_{s=v-1}^{b+v-2} s^{\frac{v-1}{1}}(b+v-2-t)^{\frac{v-1}{h}} \bar{h}(s)
\end{aligned}
$$




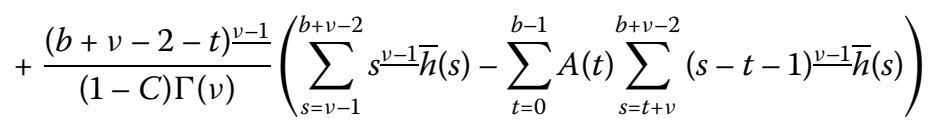

$$
\begin{aligned}
& =\sum_{s=v-1}^{b+v-2} G(t, s) h(s-v+1)+\frac{(b+v-2-t)^{\frac{v-1}{2}}}{(1-C) \Gamma(v)} \\
& \times\left(C \sum_{s=\nu-1}^{b+\nu-2} s \frac{\nu-1}{h}(s)-\sum_{t=0}^{b-1} A(t) \sum_{s=t+v}^{b+\nu-2}(s-t-1)^{\frac{\nu-1}{h}} \bar{h}(s)\right) \\
& =\sum_{s=v-1}^{b+v-2} G(t, s) h(s-v+1)+\frac{(b+v-2-t)^{\frac{\nu-1}{u}}}{(1-C) \Gamma(v)} \\
& \times\left(\sum_{t=0}^{b-1} \sum_{s=v-1}^{b+v-2} \frac{s^{\frac{\nu-1}{}}(b+v-2-t)^{\frac{\nu-1}{2}} A(t) \bar{h}(s)}{(b+v-1)^{\underline{\nu-1}}}-\sum_{t=0}^{b-1} A(t) \sum_{s=t+\nu}^{b+v-2}(s-t-1)^{\frac{\nu-1}{h}} \bar{h}(s)\right) \\
& =\sum_{s=v-1}^{b+v-2} G(t, s) h(s-v+1)+\frac{(b-2+v-t)^{\nu-1}}{(1-C) \Gamma(v)} \sum_{s=\nu-1}^{b+v-2} \sum_{t=0}^{b-1} G(t, s) A(t) \bar{h}(s) \\
& =\sum_{s=v-1}^{b+v-2} J(t, s) h(s-v+1)
\end{aligned}
$$

The proof is complete.

Lemma 2.9 Let $0 \leq C<1$ and $h:[0, b]_{\mathbb{N}_{0}} \rightarrow \mathbb{R}$. Problem (2.5) is equivalent to the following problem:

$$
\left\{\begin{array}{l}
{ }_{b+\varepsilon-1} \nabla^{v-\varepsilon} y(t)+h(t+v-1)=0, \quad t \in[v-1, b+v-1]_{\mathbb{N}_{v-1}}, \\
y(b+\varepsilon)=0, \quad\left[b+\varepsilon-1 \nabla^{-\varepsilon} y(t)\right]_{-1}=\sum_{t=0}^{b-1}\left(b+\varepsilon-1 \nabla^{-\varepsilon} y(t)\right) A(t) .
\end{array}\right.
$$

Proof Suppose that $x(t)$ is a solution of (2.5). Let

$$
y(t)={ }_{b} \nabla^{\varepsilon} x(t) .
$$

Then by Lemma 2.7 and $x(b)=0$, we have $x(t)={ }_{b+\varepsilon-1} \nabla^{-\varepsilon} y(t)$.

By Lemma 2.3, we get

$$
{ }_{b} \nabla^{v} x(t)={ }_{b-1} \nabla^{v} x(t)={ }_{b-1} \nabla_{b+\varepsilon-1}^{v} \nabla^{-\varepsilon} y(t)={ }_{b+\varepsilon-1} \nabla^{\nu-\varepsilon} y(t) .
$$

Therefore

$$
{ }_{b+\varepsilon-1} \nabla^{v-\varepsilon} y(t)+h(t+v-1)=0,
$$

and

$$
x(b)=y(b+\varepsilon)=0, \quad x(-1)=\left[{ }_{b+\varepsilon-1} \nabla^{-\varepsilon} y(t)\right]_{-1}=\sum_{t=0}^{b-1}\left({ }_{b+\varepsilon-1} \nabla^{-\varepsilon} y(t)\right) A(t) .
$$

Same if vice versa.

Using Lemmas 2.8 and 2.9, we may easily obtain the following Lemmas 2.10 and 2.11 . 
Lemma 2.10 Let $0 \leq C<1$, problem (1.1) and (1.2) is equivalent to the following problem:

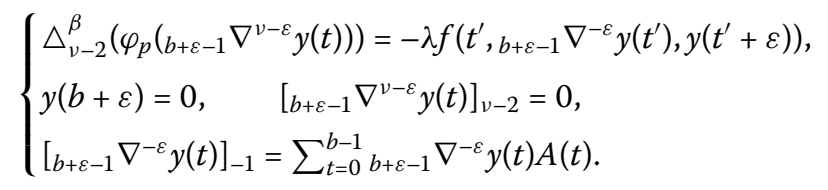

Lemma 2.11 FBVP (2.7) has the unique solution

$$
y(t)=\sum_{s=\nu-1}^{b+\nu-2} \bar{J}(t, s) \varphi_{q}\left(\lambda \triangle_{\nu-\beta-1}^{-\beta} f\left(s^{\prime},{ }_{b+\varepsilon-1} \nabla^{-\varepsilon} y\left(s^{\prime}\right), y\left(s^{\prime}+\varepsilon\right)\right)\right) .
$$

Conversely, if $y(t)$ satisfies (2.8), then $y(t)$ is a solution of $(2.7)$, where $\bar{J}(t, s)$ is given by (2.4).

Lemma 2.12 The function $\bar{J}(t, s)$ has the following properties:

(i) $\bar{J}(t, s) \geq 0,(t, s) \in[\varepsilon, b+\varepsilon]_{\mathbb{N}_{\varepsilon}} \times[v-1, b+v-1]_{\mathbb{N}_{v-1}}$,

(ii) $(b+v-2-t) \frac{v-\varepsilon-1}{m} m(s) \leq \bar{J}(t, s) \leq M(b+v-2-t)^{\frac{\nu-\varepsilon-1}{}}, t \in[\varepsilon, b+\varepsilon]_{\mathbb{N}_{\varepsilon}}$, where $\bar{J}(t, s)$ is given by (2.4), and

$$
\begin{aligned}
& m(s)=\frac{G_{A}(s)}{(1-C) \Gamma(v-\varepsilon)}, \\
& M=1+\frac{\left\|G_{A}\right\|}{(1-C) \Gamma(v-\varepsilon)}, \quad\left\|G_{A}\right\|=\max _{s \in[v-1, b+v-1]_{\mathbb{N}-1}}\left|G_{A}(s)\right| .
\end{aligned}
$$

The proof of (i) is similar to Theorem 3.2 of [18], hence it is omitted. For (ii), we note that

$$
\begin{aligned}
& \bar{J}(t, s) \leq \frac{s^{\frac{\nu-1}{}}(b+v-2-t)^{\frac{\nu-\varepsilon-1}{}}}{(b+v-1)^{\underline{\nu-1}}}+\frac{\left\|G_{A}\right\|(b+v-2-t)^{\nu-\varepsilon-1}}{(1-C) \Gamma(v-\varepsilon)} \\
& \leq\left(1+\frac{\left\|G_{A}\right\|}{(1-C) \Gamma(v-\varepsilon)}\right)(b+v-2-t)^{\frac{v-\varepsilon-1}{2}} .
\end{aligned}
$$

Then it is easy to get properties (ii).

Definition 2.3 A function $\phi(t)$ is called a lower solution of (2.7) if it satisfies

$$
\left\{\begin{array}{l}
-\triangle_{\nu-2}^{\beta}\left(\varphi_{p}\left(b_{b+\varepsilon-1} \nabla^{\nu-\varepsilon} \phi(t)\right)\right) \leq \lambda f\left(t^{\prime},{ }_{b+\varepsilon-1} \nabla^{-\varepsilon} \phi\left(t^{\prime}\right), \phi\left(t^{\prime}+\varepsilon\right)\right) \\
\phi(b+\varepsilon) \geq 0, \quad\left[{ }_{b+\varepsilon-1} \nabla^{\nu-\varepsilon} \phi(t)\right]_{\nu-2} \geq 0 \\
{\left[{ }_{b+\varepsilon-1} \nabla^{-\varepsilon} \phi(t)\right]_{-1} \geq \sum_{t=0}^{b-1}\left({ }_{b+\varepsilon-1} \nabla^{-\varepsilon} \phi(t)\right) A(t)}
\end{array}\right.
$$

Definition 2.4 A function $\psi(t)$ is called an upper solution of (2.7) if it satisfies

$$
\left\{\begin{array}{l}
\left.-\triangle_{\nu-2}^{\beta}\left(\varphi_{p}\left(b+\varepsilon-1 \nabla^{\nu-\varepsilon} \psi(t)\right)\right) \geq \lambda f\left(t^{\prime},{ }_{b+\varepsilon-1} \nabla^{-\varepsilon} \psi\left(t^{\prime}\right)\right), \psi\left(t^{\prime}+\varepsilon\right)\right), \\
\psi(b+\varepsilon) \leq 0, \quad\left[{ }_{b+\varepsilon-1} \nabla^{\nu-\varepsilon} \psi(t)\right]_{v-2} \leq 0 \\
{\left[{ }_{b+\varepsilon-1} \nabla^{-\varepsilon} \psi(t)\right]_{-1} \leq \sum_{t=0}^{b-1}\left({ }_{b+\varepsilon-1} \nabla^{-\varepsilon} \psi(t)\right) A(t) .}
\end{array}\right.
$$


Remark 2.2 Assume $0 \leq C<1, G_{A}(s) \geq 0$ for $s \in[v-1, b+v-1]_{\mathbb{N}_{v-1}}$, and $y:[\varepsilon$, $b+\varepsilon]_{\mathbb{N}_{\varepsilon}} \rightarrow \mathbb{R}$ with

$$
\begin{aligned}
& y(b+\varepsilon)=0, \quad\left[{ }_{b+\varepsilon-1} \nabla^{-\varepsilon} y(t)\right]_{-1}=\sum_{t=0}^{b-1}\left({ }_{b+\varepsilon-1} \nabla^{-\varepsilon} y(t)\right) A(t), \\
& -{ }_{b+\varepsilon-1} \nabla^{\nu-\varepsilon} y(t) \geq 0, \quad t \in[v, b+v]_{\mathbb{N}_{v}} .
\end{aligned}
$$

Then $y(t) \geq 0, t \in[\varepsilon, b+\varepsilon]_{\mathbb{N}_{\varepsilon}}$.

In fact, let ${ }_{b+\varepsilon-1} \nabla^{\nu-\varepsilon} y(t)=\eta(t)$. Then $y(t)=\sum_{s=\nu-1}^{b+\nu-2} \bar{J}(t, s) \eta(s)$.

From $\eta(t) \geq 0$, we can get the conclusion $y(t) \geq 0, t \in[\varepsilon, b+\varepsilon]_{\mathbb{N}_{\varepsilon}}$.

Lemma 2.13 (Schauder fixed point theorem) Let $T$ be a continuous and compact mapping of a Banach space $\mathbb{E}$ into itself such that the set

$$
\{x \in \mathbb{E}: x=\sigma T x\}
$$

for some $0 \leq \sigma \leq 1$ is bounded. Then $T$ has a fixed point.

\section{The method of upper and lower solutions}

To establish the existence of a solution for the boundary value problem, we need to make the following assumptions.

$\left(H_{4}\right) A$ is defined on $[0, b]_{\mathbb{N}_{0}}$, satisfying $G_{A}(s) \geq 0$ for $s \in[v-1, b+v-1]_{\mathbb{N}_{v-1}}$, and $0 \leq$ $C<1$.

$\left(H_{5}\right) f(\cdot, u, s):[0, b]_{\mathbb{N}_{0}} \times[0,+\infty) \times[0,+\infty) \rightarrow[0,+\infty)$ is continuous and is nonincreasing on $u$ and $s$. For all $\lambda \in(0,1)$, there exist two constants $\mu_{1}, \mu_{2}>0$ such that, for any $(t, u, s) \in[0, b]_{\mathbb{N}_{0}} \times[0,+\infty) \times[0,+\infty)$,

$$
\begin{aligned}
& f(t, \lambda u, s) \leq \lambda^{-\mu_{1}} f(t, u, s), \\
& f(t, u, \lambda s) \leq \lambda^{-\mu_{2}} f(t, u, s) .
\end{aligned}
$$

Remark 3.1 Inequalities (3.1), (3.2) are equivalent to the following inequalities (3.3), (3.4), respectively:

$$
\begin{array}{ll}
f(t, \lambda u, s) \geq \lambda^{-\mu_{1}} f(t, u, s), & \forall \lambda>1, \\
f(t, u, \lambda s) \geq \lambda^{-\mu_{2}} f(t, u, s), \quad \forall \lambda>1 .
\end{array}
$$

Now we denote

$$
\xi=\frac{l_{y}^{-\left(\mu_{1}+\mu_{2}\right)}}{\Gamma(\beta)}, \quad \zeta=\frac{l_{y}^{\mu_{1}+\mu_{2}}}{\Gamma(\beta)}
$$

and $g(t)=f\left(t^{\prime},{ }_{b+\varepsilon-1} \nabla^{-\varepsilon}\left(t^{\prime \prime}\right)^{\frac{\nu-\varepsilon-1}{}},\left(t^{\prime \prime}-\varepsilon\right)^{\frac{\nu-\varepsilon-1}{2}}\right), t \in \mathbb{T}$. Then $g(t) \in C(\mathbb{T}, \mathbb{R})$, for $m \in(0,1)$, we define

$$
\|g\|_{\frac{1}{m}}:=\left(\sum_{s=\nu-\beta-1}^{b+\nu-\beta-2} g^{\frac{1}{m}}(s)\right)^{m} .
$$


Theorem 3.1 Suppose that $\left(H_{4}\right)$ and $\left(H_{5}\right)$ hold. Then there exists a constant $\lambda^{*}>0$ such that FBVP (2.7) has at least one positive solution $w(t)$ for any $\lambda \in\left(\lambda^{*},+\infty\right)$. Moreover, there exists a constant $0<l<1$ such that

$$
l(b+v-2-t)^{\frac{v-\varepsilon-1}{} \leq w(t)} \leq l^{-1}(b+v-2-t)^{\frac{v-\varepsilon-1}{} .}
$$

Proof Let $Q=C\left([\varepsilon, b+\varepsilon]_{\mathbb{N}_{\varepsilon}}, \mathbb{R}\right)$, and define a subset $P$ of $Q$ as follows:

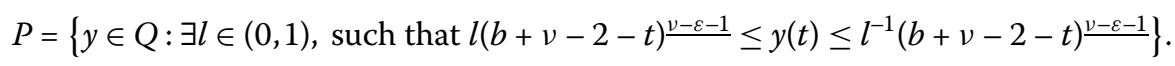

Clearly, $P$ is a nonempty set since $(b+v-2-t) \frac{\nu-\varepsilon-1}{\underline{ }} \in P$. Now define the operator $T_{\lambda}$ in $P$.

$$
T_{\lambda} y(t)=\sum_{s=\nu-1}^{b+\nu-2} \bar{J}(t, s) \varphi_{q}\left(\triangle_{\nu-\beta-1}^{-\beta} \lambda f\left(s^{\prime}{ }_{b+\varepsilon-1} \nabla^{-\varepsilon} y\left(s^{\prime}\right), y\left(s^{\prime}+\varepsilon\right)\right)\right),
$$

where $\bar{J}(t, s)$ is given by (2.4).

We assert that $T_{\lambda}$ is well defined and $T_{\lambda}(P) \subset P$.

In fact, for any $y \in P$, there exists a positive number $0<l_{y}<1$ such that

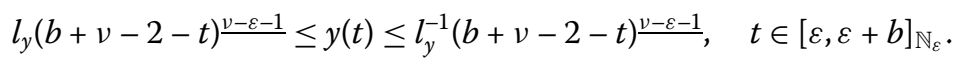

Thus, by Lemma 2.12, condition $\left(H_{5}\right)$, Hölder's inequality and noticing $m \in(0,1)$, we get

$$
\begin{aligned}
& T_{\lambda} y(t)=\sum_{s=\nu-1}^{b+\nu-2} \bar{J}(t, s) \varphi_{q}\left(\triangle_{\nu-\beta-1}^{-\beta} \lambda f\left(s^{\prime},{ }_{b+\varepsilon-1} \nabla^{-\varepsilon} y\left(s^{\prime}\right), y\left(s^{\prime}+\varepsilon\right)\right)\right) \\
& \leq \lambda^{q-1} \sum_{s=\nu-1}^{b+\nu-2} \bar{J}(t, s) \varphi_{q}\left(\triangle_{\nu-\beta-1}^{-\beta} f\left(s^{\prime},{ }_{b+\varepsilon-1} \nabla^{-\varepsilon} l_{y}\left(s^{\prime \prime}\right)^{\frac{\nu-\varepsilon-1}{}}, l_{y}\left(s^{\prime \prime}-\varepsilon\right)^{\frac{\nu-\varepsilon-1}{}}\right)\right) \\
& \leq \lambda^{q-1} \sum_{s=\nu-1}^{b+\nu-2} \bar{J}(t, s) \varphi_{q}\left(\xi \sum_{u=\nu-\beta-1}^{s-\beta}(s-u-1)^{\frac{\beta-1}{g}} g(u)\right) \\
& \left.\leq \sum_{s=\nu-1}^{b+\nu-2} \lambda^{q-1} M(b+v-2-t)^{\nu-\varepsilon-1} \xi^{q-1}\left(\sum_{u=\nu-\beta-1}^{s-\beta}(s-u-1)\right)^{\beta-1} g(u)\right)^{q-1} \\
& \leq(\xi \lambda)^{q-1} M(b+\nu-2-\varepsilon)^{\frac{\nu-\varepsilon-1}{}}\|g\|_{\frac{1}{m}}^{q-1} \\
& \times \sum_{s=\nu-1}^{b+\nu-2}\left(\sum_{u=\nu-\beta-1}^{s-\beta}\left((s-u-1)^{\frac{\beta-1}{2}}\right)^{\frac{1}{1-m}}\right)^{(1-m)(q-1)} \\
& <+\infty \text {, }
\end{aligned}
$$

i.e.,

$$
T_{\lambda} y(t)<+\infty
$$


On the other hand, using Lemma 2.12 and Remark 3.1, we have

$$
\begin{aligned}
& T_{\lambda} y(t)=\sum_{s=\nu-1}^{b+\nu-2} \bar{J}(t, s) \varphi_{q}\left(\triangle_{\nu-\beta-1}^{-\beta} \lambda f\left(s^{\prime},_{b+\varepsilon-1} \nabla^{-\varepsilon} y\left(s^{\prime}\right), y\left(s^{\prime}+\varepsilon\right)\right)\right) \\
& \geq \lambda^{q-1} \sum_{s=\nu-1}^{b+v-2} \bar{J}(t, s) \varphi_{q}\left(\triangle_{\nu-\beta-1}^{-\beta} f\left(s^{\prime},_{b+\varepsilon-1} \nabla^{-\varepsilon} l_{y}^{-1}\left(s^{\prime \prime}\right)^{\frac{v-\varepsilon-1}{}}, l_{y}^{-1}\left(s^{\prime \prime}-\varepsilon\right)^{\frac{v-\varepsilon-1}{}}\right)\right) \\
& \geq \lambda^{q-1} \sum_{s=\nu-1}^{b+v-2} \bar{J}(t, s) \varphi_{q}\left(\zeta \sum_{u=v-\beta-1}^{s-\beta}(s-u-1) \frac{\beta-1}{g} g(u)\right) \\
& \geq(\lambda \zeta)^{q-1} \sum_{s=v-1}^{b+v-2} m(s)(b+v-2-t)^{\frac{v-\varepsilon-1}{}} \varphi_{q}\left(\sum_{u=v-\beta-1}^{s-\beta}(s-u-1) \frac{\beta-1}{g} g(u)\right)
\end{aligned}
$$

Therefore

$$
T_{\lambda} y(t) \geq(\lambda \zeta)^{q-1}(b+v-2-t)^{\frac{\nu-\varepsilon-1}{b+\nu-2}} \sum_{s=\nu-1}^{b} m(s)\left(\sum_{u=\nu-\beta-1}^{s-\beta}(s-u-1) \frac{\beta-1}{g(u)}\right)^{q-1}
$$

Choose

$$
\begin{aligned}
I_{y}= & \min \left\{\frac{1}{2},\left[(\lambda \xi)^{q-1} M\|g\|_{\frac{1}{m}}^{q-1} \sum_{s=\nu-1}^{b+v-2}\left(\sum_{u=\nu-\beta-1}^{s-\beta}\left((s-u-1)^{\beta-1}\right)^{\frac{1}{1-m}}\right)^{(1-m)(q-1)}\right]^{-1},\right. \\
& (\lambda \zeta)^{q-1} \sum_{s=\nu-1}^{b+\nu-2} m(s)\left(\sum_{u=\nu-\beta-1}^{s-\beta}(s-u-1)^{\left.\left.\frac{\beta-1}{2} g(u)\right)^{q-1}\right\} .}\right.
\end{aligned}
$$

Then it follows from (3.6), (3.7) and (3.8) that

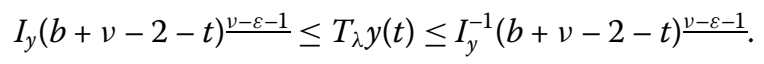

Next we shall devote our attention to finding the upper and lower solutions of FBVP (2.7). Let

$$
e(t)=\sum_{s=v-1}^{b+\nu-2} \bar{J}(t, s) \varphi_{q}\left(\triangle_{\nu-\beta-1}^{-\beta} g(s)\right)
$$

By Lemma 2.12, we have

$$
e(t) \geq(b+v-2-t)^{\frac{v-\varepsilon-1}{b+\nu-2}} \sum_{s=v-1} m(s) \varphi_{q}\left(\triangle_{\nu-\beta-1}^{-\beta} g(s)\right), \quad \forall t \in[\varepsilon, b+\varepsilon]_{\mathbb{N}_{\varepsilon}},
$$

and consequently there exists a constant $\lambda_{1} \geq 1$ such that

$$
\lambda_{1} e(t) \geq(b+v-2-t)^{\frac{v-\varepsilon-1}{}} .
$$


Thus, for any $\lambda>\lambda_{1}$, by $\left(H_{5}\right)$ and similar to (3.6), we have

$$
\begin{aligned}
& \sum_{s=\nu-1}^{b+\nu-2} \bar{J}(t, s) \varphi_{q}\left(\triangle_{\nu-\beta-1}^{-\beta} f\left(s^{\prime},_{b+\varepsilon-1} \nabla^{-\varepsilon} \lambda e\left(s^{\prime}\right), \lambda e\left(s^{\prime}+\varepsilon\right)\right)\right) \\
& \leq \sum_{s=\nu-1}^{b+\nu-2} \bar{J}(t, s) \varphi_{q}\left(\triangle_{\nu-\beta-1}^{-\beta} f\left(s^{\prime},_{b+\varepsilon-1} \nabla^{-\varepsilon} \lambda_{1} e\left(s^{\prime}\right), \lambda_{1} e\left(s^{\prime}+\varepsilon\right)\right)\right) \\
& \leq \sum_{s=\nu-1}^{b+\nu-2} \bar{J}(t, s) \varphi_{q}\left[\sum_{u=\nu-\beta-1}^{s-\beta}\left(\frac{(s-u-1)^{\beta-1}}{\Gamma(\beta)} f\left(u^{\prime},\left({ }_{b+\varepsilon-1} \nabla^{-\varepsilon}\left(u^{\prime \prime}\right)^{\frac{\nu-\varepsilon-1}{}}\right),\left(u^{\prime \prime}-\varepsilon\right)^{\frac{\nu-\varepsilon-1}{}}\right)\right)\right] \\
& =\sum_{s=\nu-1}^{b+\nu-2} \bar{J}(t, s) \varphi_{q}\left[\sum_{u=\nu-\beta-1}^{s-\beta}\left(\frac{(s-u-1)^{\frac{\beta-1}{}}}{\Gamma(\beta)} g(u)\right)\right] \\
& <+\infty \text {, }
\end{aligned}
$$

and

$$
e(t) \leq M(b+v-2-t)^{\frac{v-\varepsilon-1}{b+v-2}} \sum_{s=v-1} \varphi_{q}\left(\sum_{u=v-\beta-1}^{s-\beta} \frac{1}{\Gamma(\beta)}(s-u-1)^{\beta-1} g(u)\right)<+\infty .
$$

Now let

$$
\rho=M(b+\nu-2-\varepsilon)^{\frac{\nu-\varepsilon-1}{}}\left(\frac{1}{\Gamma(\beta)}\right)^{q-1}\|g\|_{\frac{1}{m}}^{q-1} \sum_{s=\nu-1}^{b+\nu-2}\left(\sum_{u=\nu-\beta-1}^{s-\beta}\left((s-u-1)^{\frac{\beta-1}{1}}\right)^{\frac{1}{1-m}}\right)^{(1-m)(q-1)}
$$

+1 .

Take

$$
\begin{aligned}
\lambda^{*}= & \max \left\{\lambda_{1}^{\frac{1}{q-1}},\right. \\
& {\left.\left[\rho^{-\left(\mu_{1}+\mu_{2}\right)(q-1)} \sum_{s=\nu-1}^{b+v-2} m(s) \varphi_{q}\left(\triangle_{\nu-\beta-1}^{-\beta} f\left(s^{\prime},{ }_{b+\varepsilon-1} \nabla^{-\varepsilon} 1,1\right)\right)\right]^{\frac{1}{\left.\left(\mu_{1}+\mu_{2}\right)(q-1)-1\right](q-1)}}\right\} . }
\end{aligned}
$$

Then by Lemma 2.12, (3.3) and (3.4), for $\forall t \in[\varepsilon, b+\varepsilon]_{\mathbb{N}_{\varepsilon}}$, we can get

$$
\begin{aligned}
& +\infty>\sum_{s=\nu-1}^{b+v-2} \bar{J}(t, s) \varphi_{q}\left(\triangle_{\nu-\beta-1}^{-\beta} \lambda^{*} f\left(s^{\prime}{ }_{b+\varepsilon-1} \nabla^{-\varepsilon}\left(\lambda^{*}\right)^{q-1} e\left(s^{\prime}\right),\left(\lambda^{*}\right)^{q-1} e\left(s^{\prime}+\varepsilon\right)\right)\right) \\
& \geq(b+v-2-t)^{\frac{\nu-\varepsilon-1}{}\left(\lambda^{*}\right)^{\left[1-\left(\mu_{1}+\mu_{2}\right)(q-1)\right](q-1)}} \\
& \times \sum_{s=\nu-1}^{b+\nu-2} m(s) \varphi_{q}\left(\triangle_{\nu-\beta-1}^{-\beta} f\left(s^{\prime},{ }_{b+\varepsilon-1} \nabla^{-\varepsilon} e\left(s^{\prime}\right), e\left(s^{\prime}+\varepsilon\right)\right)\right) \\
& \geq(b+v-2-t)^{\frac{v-\varepsilon-1}{}}\left(\lambda^{*}\right)^{\left[1-\left(\mu_{1}+\mu_{2}\right)(q-1)\right](q-1)} \sum_{s=\nu-1}^{b+v-2} m(s) \varphi_{q}\left(\triangle_{\nu-\beta-1}^{-\beta} f\left(s^{\prime},_{b+\varepsilon-1} \nabla^{-\varepsilon} \rho, \rho\right)\right) \\
& \geq(b+v-2-t)^{\frac{v-\varepsilon-1}{}}\left(\lambda^{*}\right)^{\left[1-\left(\mu_{1}+\mu_{2}\right)(q-1)\right](q-1)} \rho^{-\left(\mu_{1}+\mu_{2}\right)(q-1)}
\end{aligned}
$$




$$
\begin{aligned}
& \times \sum_{s=\nu-1}^{b+\nu-2} m(s) \varphi_{q}\left(\triangle_{\nu-\beta-1}^{-\beta} f\left(s^{\prime},{ }_{b+\varepsilon-1} \nabla^{-\varepsilon} 1,1\right)\right) \\
\geq & (b+v-2-t)^{\frac{\nu-\varepsilon-1}{u}} .
\end{aligned}
$$

That is to say,

$$
\begin{aligned}
& \sum_{s=\nu-1}^{b+\nu-2} \bar{J}(t, s) \varphi_{q}\left(\triangle_{\nu-\beta-1}^{-\beta} \lambda^{*} f\left(s^{\prime}{ }_{b+\varepsilon-1} \nabla^{-\varepsilon}\left(\lambda^{*}\right)^{q-1} e\left(s^{\prime}\right),\left(\lambda^{*}\right)^{q-1} e\left(s^{\prime}+\varepsilon\right)\right)\right) \\
& \quad \geq(b+v-2-t)^{\frac{\nu-\varepsilon-1}{}} .
\end{aligned}
$$

Let

$$
\phi(t)=\left(\lambda^{*}\right)^{q-1} e(t)=T_{\lambda^{*}}\left((b+\nu-2-t)^{\underline{\nu-\varepsilon-1}}\right), \quad \psi(t)=T_{\lambda^{*}}(\phi(t)) .
$$

It follows from (3.9) and (3.10) that for any $t \in[\varepsilon, b+\varepsilon]_{\mathbb{N}_{\varepsilon}}$,

$$
\left\{\begin{array}{l}
\phi(t)=\sum_{s=v-1}^{b+\nu-2} \bar{J}(t, s) \varphi_{q}\left(\triangle_{\nu-\beta-1}^{-\beta} \lambda^{*} g(s)\right) \geq \lambda_{1} e(t) \geq(b+v-2-t)^{\nu-\varepsilon-1} \\
\psi(t)=\sum_{s=\nu-1}^{b+\nu-2} \bar{J}(t, s) \varphi_{q}\left(\triangle_{\nu-\beta-1}^{-\beta} \lambda^{*} f\left(s^{\prime},{ }_{b+\varepsilon-1} \nabla^{-\varepsilon}\left(\lambda^{*}\right)^{q-1} e\left(s^{\prime}\right),\left(\lambda^{*}\right)^{q-1} e\left(s^{\prime}+\varepsilon\right)\right)\right) .
\end{array}\right.
$$

Moreover, by (3.11) and (3.12), we know

$$
\left\{\begin{array}{l}
\phi(b+\varepsilon)=0, \quad\left[{ }_{b+\varepsilon-1} \nabla^{\nu-\varepsilon} \phi(t)\right]_{\nu-2}=0, \\
{\left[{ }_{b+\varepsilon-1} \nabla^{-\varepsilon} \phi(t)\right]_{-1}=\sum_{t=0}^{b-1}\left({ }_{b+\varepsilon-1} \nabla^{-\varepsilon} \phi(t)\right) A(t),} \\
\psi(b+\varepsilon)=0, \quad\left[{ }_{b+\varepsilon-1} \nabla^{v-\varepsilon} \psi(t)\right]_{v-2}=0, \\
{\left[{ }_{b+\varepsilon-1} \nabla^{-\varepsilon} \psi(t)\right]_{-1}=\sum_{t=0}^{b-1}\left({ }_{b+\varepsilon-1} \nabla^{-\varepsilon} \psi(t)\right) A(t) .}
\end{array}\right.
$$

Proceeding as in (3.6)-(3.8), we get that $\phi(t), \psi(t) \in P$. By (3.10), we have

$$
\psi(t)=\left(T_{\lambda^{*}} \phi\right)(t) \geq(b+v-2-t)^{\frac{\nu-\varepsilon-1}{}},
$$

which implies

$$
\begin{aligned}
\psi(t) & =\left(T_{\lambda^{*}} \phi\right)(t) \\
& =\sum_{s=\nu-1}^{b+v-2} \bar{J}(t, s) \varphi_{q}\left(\triangle_{\nu-\beta-1}^{-\beta} \lambda^{*} f\left(s^{\prime}, b+\varepsilon-1 \nabla^{-\varepsilon}\left(\lambda^{*}\right)^{q-1} e\left(s^{\prime}\right),\left(\lambda^{*}\right)^{q-1} e\left(s^{\prime}+\varepsilon\right)\right)\right) \\
& \leq \sum_{s=\nu-1}^{b+\nu-2} \bar{J}(t, s) \varphi_{q}\left(\triangle_{\nu-\beta-1}^{-\beta} \lambda^{*} g(s)\right)=\phi(t), \quad \forall t \in[\varepsilon, b+\varepsilon]_{\mathbb{N}_{\varepsilon}} .
\end{aligned}
$$

Thus, taking account of $f$ being nonincreasing, and by (3.11), (3.14) and (3.15), we have

$$
\begin{aligned}
& \triangle_{v-2}^{\beta}\left(\varphi_{p}\left({ }_{b+\varepsilon-1} \nabla^{v-\varepsilon} \psi\right)\right)(t)+\lambda^{*} f\left(t^{\prime},{ }_{b+\varepsilon-1} \nabla^{-\varepsilon} \psi\left(t^{\prime}\right), \psi\left(t^{\prime}+\varepsilon\right)\right) \\
& \quad=\triangle_{v-2}^{\beta}\left(\varphi_{p}\left({ }_{b+\varepsilon-1} \nabla^{v-\varepsilon}\left(T_{\lambda^{*}} \phi\right)\right)\right)(t)+\lambda^{*} f\left(t^{\prime},{ }_{b+\varepsilon-1} \nabla^{-\varepsilon} \psi\left(t^{\prime}\right), \psi\left(t^{\prime}+\varepsilon\right)\right) \\
& \geq \triangle_{v-2}^{\beta}\left(\varphi_{p}\left({ }_{b+\varepsilon-1} \nabla^{\nu-\varepsilon}\left(T_{\lambda^{*}} \phi\right)\right)\right)(t)+\lambda^{*} f\left(t^{\prime}{ }_{b+\varepsilon-1} \nabla^{-\varepsilon} \phi\left(t^{\prime}\right), \phi\left(t^{\prime}+\varepsilon\right)\right)
\end{aligned}
$$




$$
\begin{aligned}
& =-\lambda^{*} f\left(t^{\prime},{ }_{b+\varepsilon-1} \nabla^{-\varepsilon} \phi\left(t^{\prime}\right), \phi\left(t^{\prime}+\varepsilon\right)\right)+\lambda^{*} f\left(t^{\prime},{ }_{b+\varepsilon-1} \nabla^{-\varepsilon} \phi\left(t^{\prime}\right), \phi\left(t^{\prime}+\varepsilon\right)\right) \\
& =0 \text {, } \\
& \triangle_{v-2}^{\beta}\left(\varphi_{p}\left({ }_{b+\varepsilon-1} \nabla^{v-\varepsilon} \phi\right)\right)(t)+\lambda^{*} f\left(t^{\prime},{ }_{b+\varepsilon-1} \nabla^{-\varepsilon} \phi\left(t^{\prime}\right), \phi\left(t^{\prime}+\varepsilon\right)\right) \\
& =\triangle_{v-2}^{\beta}\left(\varphi_{p}\left({ }_{b+\varepsilon-1} \nabla^{v-\varepsilon}\left(T_{\lambda^{*}}\left((b+v-2-t)^{\nu-\varepsilon-1}\right)\right)\right)\right)+\lambda^{*} f\left(t^{\prime}{ }_{b+\varepsilon-1} \nabla^{-\varepsilon} \phi\left(t^{\prime}\right), \phi\left(t^{\prime}+\varepsilon\right)\right) \\
& =-\lambda^{*} f\left(t^{\prime},{ }_{b+\varepsilon-1} \nabla^{\nu-\varepsilon}\left(t^{\prime \prime}\right)^{\frac{v-\varepsilon-1}{u}},\left(t^{\prime \prime}-\varepsilon\right)^{\left.\frac{v-\varepsilon-1}{}\right)}+\lambda^{*} f\left(t^{\prime},{ }_{b+\varepsilon-1} \nabla^{-\varepsilon} \phi\left(t^{\prime}\right), \phi\left(t^{\prime}+\varepsilon\right)\right)\right. \\
& \leq-\lambda^{*} f\left(t^{\prime},{ }_{b+\varepsilon-1} \nabla^{\nu-\varepsilon}\left(t^{\prime \prime}\right)^{\frac{\nu-\varepsilon-1}{2}},\left(t^{\prime \prime}-\varepsilon\right)^{\frac{\nu-\varepsilon-1}{2}}\right) \\
& +\lambda^{*} f\left(t^{\prime},{ }_{b+\varepsilon-1} \nabla^{\nu-\varepsilon}\left(t^{\prime \prime}\right)^{\frac{\nu-\varepsilon-1}{2}},\left(t^{\prime \prime}-\varepsilon\right)^{\frac{v-\varepsilon-1}{2}}\right) \\
& =0 \text {. }
\end{aligned}
$$

It follows from (3.13) and (3.15)-(3.17) that $\psi(t), \phi(t)$ are upper and lower solutions of $\operatorname{FBVP}(2.7)$ and $\psi(t), \phi(t) \in P$.

Now we define a function

$$
F\left(t^{\prime},{ }_{b+\varepsilon-1} \nabla^{-\varepsilon} y\left(t^{\prime}\right), y\left(t^{\prime}\right)\right)= \begin{cases}f\left(t^{\prime},{ }_{b+\varepsilon-1} \nabla^{-\varepsilon} \psi\left(t^{\prime}\right), \psi\left(t^{\prime}+\varepsilon\right)\right), & y<\psi(t), \\ f\left(t^{\prime},{ }_{b+\varepsilon-1} \nabla^{-\varepsilon} y\left(t^{\prime}\right), y\left(t^{\prime}+\varepsilon\right)\right), & \psi(t) \leq y \leq \phi(t), \\ f\left(t^{\prime},{ }_{b+\varepsilon-1} \nabla^{-\varepsilon} \phi\left(t^{\prime}\right), \phi\left(t^{\prime}+\varepsilon\right)\right), & y>\phi(t) .\end{cases}
$$

It then follows from $\left(H_{5}\right)$ and $(3.18)$ that $F(t, u, s):[0, b]_{\mathbb{N}_{0}} \times[0,+\infty) \times[0,+\infty) \longrightarrow[0,+\infty)$ is continuous.

We now show that the FBVP

$$
\left\{\begin{array}{l}
\triangle_{v-2}^{\beta}\left(\varphi_{p}\left(b+\varepsilon-1 \nabla^{v-\varepsilon} y(t)\right)\right)=-\lambda^{*} F\left(t^{\prime},{ }_{b+\varepsilon-1} \nabla^{-\varepsilon} y\left(t^{\prime}\right), y\left(t^{\prime}+\varepsilon\right)\right) \\
\quad t \in[\varepsilon, b+\varepsilon]_{\mathbb{N}_{\varepsilon}} \\
y(b+\varepsilon)=0, \quad\left[{ }_{b+\varepsilon-1} \nabla^{\nu-\varepsilon} y(t)\right]_{v-2}=0 \\
{\left[{ }_{b+\varepsilon-1} \nabla^{-\varepsilon} y(t)\right]_{-1}=\sum_{t=0}^{b-1}\left({ }_{b+\varepsilon-1} \nabla^{-\varepsilon} y(t)\right) A(t)}
\end{array}\right.
$$

has a positive solution.

Define the operator $D_{\lambda^{*}}$ by

$$
\begin{aligned}
& D_{\lambda^{*}} y(t)=\sum_{s=\nu-1}^{b+\nu-2} \bar{J}(t, s) \varphi_{q}\left(\triangle_{\nu-\beta-1}^{-\beta} \lambda^{*} F\left(s^{\prime},{ }_{b+\varepsilon-1} \nabla^{-\varepsilon} y\left(s^{\prime}\right), y\left(s^{\prime}+\varepsilon\right)\right)\right), \\
& t \in[\varepsilon, b+\varepsilon]_{\mathbb{N}_{\varepsilon}} .
\end{aligned}
$$

Then $D_{\lambda^{*}}: C\left([\varepsilon, b+\varepsilon]_{\mathbb{N}_{\varepsilon}}, \mathbb{R}\right) \rightarrow C\left([\varepsilon, b+\varepsilon]_{\mathbb{N}_{\varepsilon}}, \mathbb{R}\right)$, and a fixed point of the operator $D_{\lambda^{*}}$ is a solution of FBVP (3.19).

On the other hand, from the definition of $F$ and the fact that the function $f$ is nonincreasing on the second and third variable, we obtain

$$
\begin{aligned}
f\left(t^{\prime},{ }_{b+\varepsilon-1} \nabla^{-\varepsilon} \phi\left(t^{\prime}\right), \phi\left(t^{\prime}+\varepsilon\right)\right) & \leq F\left(t^{\prime},{ }_{b+\varepsilon-1} \nabla^{-\varepsilon} y\left(t^{\prime}\right), y\left(t^{\prime}+\varepsilon\right)\right) \\
& \leq f\left(t^{\prime},{ }_{b+\varepsilon-1} \nabla^{-\varepsilon} \psi\left(t^{\prime}\right), \psi\left(t^{\prime}+\varepsilon\right)\right),
\end{aligned}
$$


provided that $\psi(t) \leq y(t) \leq \phi(t)$;

$$
F\left(t^{\prime},{ }_{b+\varepsilon-1} \nabla^{-\varepsilon} y\left(t^{\prime}\right), y\left(t^{\prime}+\varepsilon\right)\right)=f\left(t^{\prime},{ }_{b+\varepsilon-1} \nabla^{-\varepsilon} \psi\left(t^{\prime}\right), \psi\left(t^{\prime}+\varepsilon\right)\right)
$$

provided that $y(t)<\psi(t)$;

$$
F\left(t^{\prime},_{b+\varepsilon-1} \nabla^{-\varepsilon} y\left(t^{\prime}\right), y\left(t^{\prime}+\varepsilon\right)\right)=f\left(t^{\prime},{ }_{b+\varepsilon-1} \nabla^{-\varepsilon} \phi\left(t^{\prime}\right), \phi\left(t^{\prime}+\varepsilon\right)\right)
$$

provided that $y(t)>\phi(t)$. So we have

$$
\begin{aligned}
f\left(t^{\prime},{ }_{b+\varepsilon-1} \nabla^{-\varepsilon} \phi\left(t^{\prime}\right), \phi\left(t^{\prime}+\varepsilon\right)\right) & \leq F\left(t^{\prime},{ }_{b+\varepsilon-1} \nabla^{-\varepsilon} y\left(t^{\prime}\right), y\left(t^{\prime}+\varepsilon\right)\right) \\
& \leq f\left(t^{\prime},{ }_{b+\varepsilon-1} \nabla^{-\varepsilon} \psi\left(t^{\prime}\right), \psi\left(t^{\prime}+\varepsilon\right)\right) .
\end{aligned}
$$

Furthermore, by (3.13), (3.14) and (3.21), we have

$$
\begin{aligned}
f\left(t^{\prime}{ }_{b+\varepsilon-1} \nabla^{-\varepsilon} \phi\left(t^{\prime}\right), \phi\left(t^{\prime}+\varepsilon\right)\right) & \leq f\left(t^{\prime}{ }_{b+\varepsilon-1} \nabla^{-\varepsilon} \psi\left(t^{\prime}\right), \psi\left(t^{\prime}+\varepsilon\right)\right) \\
& \leq f\left(t^{\prime}{ }_{b+\varepsilon-1} \nabla^{-\varepsilon}\left(t^{\prime \prime}\right)^{\left.\frac{v-\varepsilon-1}{},\left(t^{\prime \prime}-\varepsilon\right)^{\frac{\nu-\varepsilon-1}{}}\right)}\right. \\
& =g(t) .
\end{aligned}
$$

It follows from Lemma 2.12 and (3.22) that for any $y \in P$,

$$
\begin{aligned}
& D_{\lambda^{*}} y(t)=\sum_{s=\nu-1}^{b+\nu-2} \bar{J}(t, s) \varphi_{q}\left(\triangle_{\nu-\beta-1}^{-\beta} \lambda^{*} F\left(s^{\prime},{ }_{b+\varepsilon-1} \nabla^{-\varepsilon} y\left(s^{\prime}\right), y\left(s^{\prime}+\varepsilon\right)\right)\right)
\end{aligned}
$$

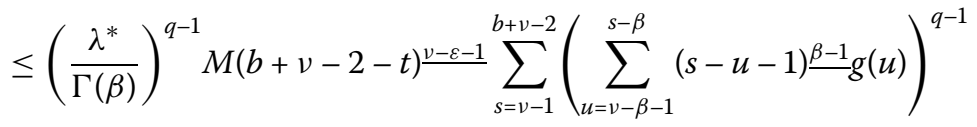

$$
\begin{aligned}
& \leq\left(\frac{\lambda^{*}}{\Gamma(\beta)}\right)^{q-1} M(b+\nu-2)^{\frac{\nu-\varepsilon-1}{n}}\|g\|_{\frac{1}{m}}^{q-1} \sum_{s=\nu-1}^{b+\nu-2} \\
& \times\left(\sum_{u=\nu-\beta-1}^{s-\beta}\left((s-u-1) \frac{\beta-1}{3}\right)^{\frac{1}{1-m}}\right)^{(1-m)(q-1)} \\
& <+\infty \text {, }
\end{aligned}
$$

namely the operator $D_{\lambda^{*}}$ is uniformly bounded.

Next, let $\Omega \subset P$ be bounded. Since the right side of (3.20) is finite sum, we can prove that $D()$ is equicontinuous. By the Arzela-Ascoli theorem, we have $D_{\lambda^{*}}: P \rightarrow P$ is completely continuous. Moreover, (3.23) implies that $D_{\lambda^{*}}$ satisfies the conditions of Lemma 2.13. Thus, by using the Schauder fixed point theorem, $D_{\lambda^{*}}$ has at least one fixed point $w$ such that $w=D_{\lambda *} w$.

Now we prove

$$
\psi(t) \leq w(t) \leq \phi(t), \quad t \in[\varepsilon, b+\varepsilon]_{\mathbb{N}_{\varepsilon}} .
$$


Since $w$ is a fixed point of $D_{\lambda^{*}}$, we have

$$
\begin{aligned}
& w(b+\varepsilon)=0, \quad\left[{ }_{b+\varepsilon-1} \nabla^{\nu-\varepsilon} w(t)\right]_{\nu-2}=0, \\
& {\left[_{b+\varepsilon-1} \nabla^{-\varepsilon} w(t)\right]_{-1}=\sum_{t=0}^{b-1}\left({ }_{b+\varepsilon-1} \nabla^{-\varepsilon} w(t)\right) A(t) .}
\end{aligned}
$$

From (3.11), (3.22) and noticing that $w$ is a fixed point of $D_{\lambda^{*}}$, we also have

$$
\begin{aligned}
& \triangle_{\nu-2}^{\beta}\left(\varphi_{p}\left({ }_{b+\varepsilon-1} \nabla^{\nu-\varepsilon} \phi(t)\right)\right)-\triangle_{v-2}^{\beta}\left(\varphi_{p}\left({ }_{b+\varepsilon-1} \nabla^{\nu-\varepsilon} w(t)\right)\right) \\
& =-\lambda^{*} f\left(t^{\prime}{ }_{b+\varepsilon-1} \nabla^{-\varepsilon}\left(t^{\prime \prime}\right)^{\frac{v-\varepsilon-1}{}},\left(t^{\prime \prime}-\varepsilon\right)^{\frac{v-\varepsilon-1}{}}\right)+\lambda^{*} F\left(t^{\prime}{ }_{b+\varepsilon-1} \nabla^{-\varepsilon} w\left(t^{\prime}\right), w\left(t^{\prime}+\varepsilon\right)\right) \\
& =-\lambda^{*} f\left(t^{\prime}{ }_{b+\varepsilon-1} \nabla^{-\varepsilon}\left(t^{\prime \prime}\right)^{\frac{\nu-\varepsilon-1}{2}},\left(t^{\prime \prime}-\varepsilon\right)^{\frac{\nu-\varepsilon-1}{}}\right)+\lambda^{*} f\left(t^{\prime}{ }_{b+\varepsilon-1} \nabla^{-\varepsilon} \psi\left(t^{\prime}\right), \psi\left(t^{\prime}+\varepsilon\right)\right) \\
& \leq-\lambda^{*} f\left(t^{\prime}{ }_{b+\varepsilon-1} \nabla^{-\varepsilon}\left(t^{\prime \prime}\right)^{\frac{\nu-\varepsilon-1}{}},\left(t^{\prime \prime}-\varepsilon\right)^{\frac{\nu-\varepsilon-1}{}}\right) \\
& +\lambda^{*} f\left(t^{\prime}{ }_{b+\varepsilon-1} \nabla^{-\varepsilon}\left(t^{\prime \prime}\right)^{\frac{\nu-\varepsilon-1}{}},\left(t^{\prime \prime}-\varepsilon\right)^{\frac{\nu-\varepsilon-1}{}}\right) \\
& =0 \text {. }
\end{aligned}
$$

Let

$$
z(t)=\varphi_{p}\left({ }_{b+\varepsilon-1} \nabla^{v-\varepsilon} \phi(t)\right)-\varphi_{p}\left({ }_{b+\varepsilon-1} \nabla^{\mathcal{v}-\varepsilon} w(t)\right)
$$

Then

$$
\begin{aligned}
& \triangle_{\nu-2}^{\beta} z(t)=\triangle_{\nu-2}^{\beta}\left(\varphi_{p}\left({ }_{b+\varepsilon-1} \nabla^{\nu-\varepsilon} \phi(t)\right)\right)-\triangle_{\nu-2}^{\beta}\left(\varphi_{p}\left({ }_{b+\varepsilon-1} \nabla^{\nu-\varepsilon} w(t)\right)\right) \leq 0, \quad t \in[\varepsilon, b+\varepsilon]_{\mathbb{N}_{\varepsilon}}, \\
& z(v-2)=\varphi_{p}\left({ }_{b+\varepsilon-1} \nabla^{\mathcal{v}-\varepsilon} \phi(v-2)\right)-\varphi_{p}\left({ }_{b+\varepsilon-1} \nabla^{\mathcal{v}-\varepsilon} w(v-2)\right)=0 .
\end{aligned}
$$

Moreover, we have $z(t) \leq 0$, i.e., $\varphi_{p}\left(b_{b+\varepsilon-1} \nabla^{v-\varepsilon} \phi(t)\right)-\varphi_{p}\left({ }_{b+\varepsilon-1} \nabla^{v-\varepsilon} w(t)\right) \leq 0$.

In fact, if we denote that

$$
\triangle_{v-2}^{\beta} z(t)=-\eta(t) \leq 0
$$

according to Lemma 2.6, we have

$$
\begin{aligned}
& z(t)=-\triangle_{v-\beta-1}^{-\beta} \eta(t)+K_{1}(t-v+\beta+1) \frac{\beta-1}{,}, \\
& z(v-2)=0 .
\end{aligned}
$$

In view of $K_{1}=0$, hence $z(t) \leq 0$.

Noticing that $\varphi_{p}$ is monotone increasing and ${ }_{b+\varepsilon-1} \nabla^{v-\varepsilon}$ is a linear operator, we have

$$
{ }_{b+\varepsilon-1} \nabla^{\mathcal{v}-\varepsilon}(\phi-w)(t) \leq 0
$$

It follows from Remark 2.2 and (3.24) that

$$
\phi(t)-w(t) \geq 0
$$


Thus we have $w(t) \leq \phi(t)$ for $t \in[\varepsilon, b+\varepsilon]_{\mathbb{N}_{\varepsilon}}$. In the same way, we also have $w(t) \geq \psi(t)$ for $t \in[\varepsilon, b+\varepsilon]_{\mathbb{N}_{\varepsilon}}$, so

$$
\psi(t) \leq w(t) \leq \phi(t), \quad t \in[\varepsilon, b+\varepsilon]_{\mathbb{N}_{\varepsilon}} .
$$

Consequently,

$$
F\left(t^{\prime},_{b+\varepsilon-1} \nabla^{-\varepsilon} w\left(t^{\prime}\right), w\left(t^{\prime}+\varepsilon\right)\right)=f\left(t^{\prime},{ }_{b+\varepsilon-1} \nabla^{-\varepsilon} w\left(t^{\prime}\right), w\left(t^{\prime}+\varepsilon\right)\right), \quad t \in[\varepsilon, b+\varepsilon]_{\mathbb{N}_{\varepsilon}} .
$$

Hence $w(t)$ is a positive solution of FBVP (3.19), i.e., $y(t){ }_{b+\varepsilon-1} \nabla^{-\varepsilon} w(t)$ is a positive solution of problem (1.1) and (1.2).

Finally, by (3.25) and $\phi, \psi \in P$, we have

$$
l_{\psi}(b+v-2-t)^{\frac{v-\varepsilon-1}{2}} \leq \psi(t) \leq w(t) \leq \phi(t) \leq l_{\phi}^{-1}(b+v-2-t)^{\frac{\nu-\varepsilon-1}{\underline{\nu}}} .
$$

Let $l_{y}=\min \left\{l_{\psi}, l_{\phi}\right\}$, then

$$
l_{y}(b+v-2-t)^{\frac{v-\varepsilon-1}{}} \leq \psi(t) \leq w(t) \leq \phi(t) \leq l_{y}^{-1}(b+v-2-t)^{\underline{v-\varepsilon-1}} .
$$

\section{Iteration of positive solutions}

To study the iteration of positive solutions to FBVP (1.1) and (1.2), we need the following assumption.

( $\left.H_{6}\right) f(\cdot, u, s):[0, b]_{\mathbb{N}_{0}} \times[0,+\infty) \times[0,+\infty) \rightarrow[0,+\infty)$ is continuous, and there exist two constants $r_{1}, r_{2}>0$ such that for any $t \in[0, b]_{\mathbb{N}_{0}}, u, s \in[0,+\infty)$.

$$
\begin{aligned}
& f(t, \lambda u, s) \geq \lambda^{r_{1}} f(t, u, s), \quad \forall \lambda \in(0,1), \\
& f(t, u, \lambda s) \geq \lambda^{r_{2}} f(t, u, s), \quad \forall \lambda \in(0,1) .
\end{aligned}
$$

Remark 4.1 Inequalities (4.1), (4.2) are equivalent to the following inequalities, respectively:

$$
\begin{array}{ll}
f(t, \lambda u, s) \leq \lambda^{r_{1}} f(t, u, s), \quad \forall \lambda>1, \\
f(t, u, \lambda s) \leq \lambda^{r_{2}} f(t, u, s), \quad \forall \lambda>1 .
\end{array}
$$

Definition 4.1 ([7]) Let $\mathbb{E}$ be a real Banach space. Let $P$ be a nonempty, convex closed set in $\mathbb{E}$. We say that $P$ is a cone if it satisfies the following properties:

(i) $\lambda u \in P$ for $u \in P, \lambda \geq 0$;

(ii) $u,-u \in P$ implies $u=\theta$ ( $\theta$ denotes the null element of $\mathbb{E})$.

Let the Banach space $\mathbb{E}=C[\varepsilon, \varepsilon+b]_{\mathbb{N} \varepsilon}$ be endowed with the norm

$$
\|y\|=\max \left\{\max _{t \in[\varepsilon, \varepsilon+b]_{\mathbb{N}_{\varepsilon}}}|y(t)|,\left.\max _{t \in[v, v+b]_{\mathbb{N}_{v}}}\right|_{b+\varepsilon-1} \nabla^{v-\varepsilon} y(t) \mid\right\} .
$$

In addition, $\mathbb{E}^{+}=\left\{u \in \mathbb{E} \mid u(t) \geq 0, t \in[\varepsilon, \varepsilon+b]_{\mathbb{N}_{\varepsilon}}\right\}$. 
Define the cone $P \subset \mathbb{E}$ by

$$
P=\left\{\left.y \in \mathbb{E}^{+}\right|_{b+\varepsilon-1} \nabla^{\nu-\varepsilon} y(t) \leq 0, t \in[v, v+b]_{\mathbb{N}_{v}}\right\}
$$

for any $y(t) \in \mathbb{E}^{+}, \lambda>0$. Define an operator

$$
(T y)(t)=\sum_{s=\nu-1}^{b+v-2} \bar{J}(t, s) \varphi_{q}\left(\triangle_{\nu-\beta-1}^{-\beta} \lambda f\left(s^{\prime},_{b+\varepsilon-1} \nabla^{-\varepsilon} y\left(s^{\prime}\right), y\left(s^{\prime}+\varepsilon\right)\right)\right),
$$

where $\bar{J}(t, s)$ is given by (2.4).

Lemma 4.1 Assume that $\left(H_{1}\right),\left(H_{2}\right)$ and $\left(H_{6}\right)$ hold, then the operator $T: P \rightarrow P$ is completely continuous.

Proof From $\left(H_{1}\right),\left(H_{2}\right),\left(H_{6}\right)$ and the definition of $T$, we deduce that for any $y \in P$, there is $(T y)(t) \geq 0$.

$$
{ }_{b+\varepsilon-1} \nabla^{v-\varepsilon} T y(t)=-\varphi_{q}\left(\triangle_{\nu-\beta-1}^{-\beta} \lambda f\left(t^{\prime},{ }_{b+\varepsilon-1} \nabla^{-\varepsilon} y\left(t^{\prime}\right), y\left(t^{\prime}+\varepsilon\right)\right)\right) \leq 0, \quad t \in[v, v+b]_{\mathbb{N}_{0}},
$$

which implies $T(P) \subset P$.

For convenience, we use the following notations. Let

$$
B=\max \left\{\max _{t \in[\varepsilon, \varepsilon+b]_{\mathbb{N}_{\varepsilon}}} \sum_{s=\nu-1}^{b+\nu-2} \bar{J}(t, s), 1\right\}, \quad N=B \varphi_{q}\left(\frac{1}{\Gamma(\beta+1)}(\beta+b-1)^{\underline{\beta}}\right) .
$$

We now give our results for the iteration of a positive solution for (2.7).

Theorem 4.1 Suppose that $\left(H_{1}\right)-\left(H_{3}\right)$ and $\left(H_{6}\right)$ hold. If there exists a positive constant $a>1$ such that

( $\left.H_{7}\right) f\left(t, u_{1}, s_{1}\right) \leq f\left(t, u_{2}, s_{2}\right)$ for any $t \in[0, b]_{\mathbb{N}_{0}}, 0 \leq u_{1}<u_{2} \leq a, 0 \leq s_{1}<s_{2} \leq a$,

$\left(H_{8}\right) \varphi_{p}(N) \leq \frac{\varphi_{p}(a)}{\lambda \sigma a^{r}+r_{2} k^{r 1}}$, where $k=\frac{(b+\varepsilon-1) \underline{\varepsilon}}{\Gamma(\varepsilon+1)} . r_{1}, r_{2}, \sigma$ are defined by (4.1), (4.2) and (1.3), respectively. Then FBVP (2.7) has two positive solutions $u^{*}$ and $w^{*}$ such that $0 \leq\left\|u^{*}\right\| \leq$ $a, 0 \leq\left\|w^{*}\right\| \leq a$.

Moreover,

$$
\begin{array}{ll}
\lim _{n \rightarrow \infty} u_{n}=\lim _{n \rightarrow \infty} T^{n} u_{0}=u^{*}, & \lim _{n \rightarrow \infty}{ }_{b+\varepsilon-1} \nabla^{\nu-\varepsilon} u_{n}={ }_{b+\varepsilon-1} \nabla^{\nu-\varepsilon} u^{*}, \\
\lim _{n \rightarrow \infty} w_{n}=\lim _{n \rightarrow \infty} T^{n} w_{0}=w^{*}, & \lim _{n \rightarrow \infty}{ }_{b+\varepsilon-1} \nabla^{\nu-\varepsilon} w_{n}={ }_{b+\varepsilon-1} \nabla^{\nu-\varepsilon} w^{*},
\end{array}
$$

where $u_{0}(t)=\frac{a}{B} \sum_{s=\nu-1}^{b+\nu-2} \bar{J}(t, s), w_{0}(t)=0, t \in[\varepsilon, \varepsilon+b]_{\mathbb{N}_{\varepsilon}} . T$ is defined by (4.5).

The iterative schemes in the theorem are

$$
u_{0}(t), u_{n+1}=T u_{n}=T^{n} u_{0}, \quad n=0,1,2, \ldots
$$

and

$$
w_{0}(t), w_{n+1}=T w_{n}=T^{n} w_{0}, \quad n=0,1,2, \ldots
$$


Proof Let $\bar{P}_{a}=\{u \in P: 0 \leq\|u\| \leq a\}$, we firstly prove $T \bar{P}_{a} \subset \bar{P}_{a}$.

If $u \in \bar{P}_{a}, T u \in P$, by $\left(H_{7}\right)$ we have

$$
\begin{aligned}
& 0 \leq u(t) \leq \max |u(t)|=\|u\| \leq a, \\
& 0 \leq f\left(t^{\prime}{ }_{b+\varepsilon-1} \nabla^{-\varepsilon} u, u\right) \leq f\left(t^{\prime}, k a, a\right) \leq k^{r_{1}} a^{r_{1}+r_{2}} f\left(t^{\prime}, 1,1\right)=\sigma k^{r_{1}} a^{r_{1}+r_{2}},
\end{aligned}
$$

where $k=\frac{(b+\varepsilon-1)^{\underline{\varepsilon}}}{\Gamma(\varepsilon+1)} \geq{ }_{b+\varepsilon-1} \nabla^{-\varepsilon} 1=1+\frac{1}{\Gamma(\varepsilon)} \sum_{s=t+\varepsilon+1}^{b+\varepsilon-1}(s-t-1)^{\frac{\varepsilon-1}{}}>1$.

Since

$$
\begin{aligned}
\|T u\| & =\max _{t \in[\varepsilon, \varepsilon+b]_{\mathbb{N}_{\varepsilon}}}|(T u)(t)|=\max _{t \in[\varepsilon, \varepsilon+b]_{\mathbb{N}_{\varepsilon}}}\left|\sum_{s=\nu-1}^{b+v-2} \bar{J}(t, s) \varphi_{q}\left(\triangle_{\nu-\beta-1}^{-\beta} \lambda f\left(s^{\prime},{ }_{,+\varepsilon-1} \nabla^{-\varepsilon} u, u\right)\right)\right| \\
& =\max _{t \in[\varepsilon, \varepsilon+b]_{\mathbb{N}_{\varepsilon}}} \sum_{s=\nu-1}^{b+\nu-2} \bar{J}(t, s) \varphi_{q}\left(\lambda \sigma k^{r_{1}} a^{r_{1}+r_{2}}\right) \varphi_{q}\left(\triangle_{\nu-\beta-1}^{-\beta} 1\right) \\
& \leq \varphi_{q}\left(\sigma \lambda k^{r_{1}} a^{r_{1}+r_{2}}\right) \max _{t \in[\varepsilon, \varepsilon+b]_{\mathbb{N}_{\varepsilon}}} \sum_{s=\nu-1}^{b+\nu-2} \bar{J}(t, s) \varphi_{q}\left(\frac{(\beta+b-1)^{\underline{\beta}}}{\Gamma(\beta+1)}\right) \\
& \leq a,
\end{aligned}
$$

we get $\|T u\| \leq a$. So we have shown that $T \bar{P}_{a} \subset \bar{P}_{a}$.

Let $u_{0}(t)=\frac{a}{B} \sum_{s=\nu-1}^{b+\nu-2} \bar{J}(t, s), t \in[\varepsilon, \varepsilon+b]_{\mathbb{N}_{\varepsilon}}$.

Then

$$
{ }_{b+\varepsilon-1} \nabla^{v-\varepsilon} u_{0}(t)=-\frac{a}{B} \leq 0
$$

It is easy to get

$$
\left|u_{0}(t)\right| \leq \frac{a \sum_{s=\nu-1}^{b+v-2} \bar{J}(t, s)}{\sum_{s=\nu-1}^{b+\nu-2} \bar{J}(t, s)}=a, \quad\left|b_{t+\varepsilon-1} \nabla^{\nu-\varepsilon} u_{0}(t)\right| \leq a .
$$

So $u_{0} \in \bar{P}_{a}$.

Let $u_{1}=T u_{0}$, we have $u_{1} \in \bar{P}_{a}$.

We define $u_{n+1}=T u_{n}=T^{n+1} u_{0}, n=0,1,2, \ldots$.

It follows from $T \bar{P}_{a} \subset \bar{P}_{a}$ that $u_{n} \in \bar{P}_{a}, n=0,1,2, \ldots$ Since $T$ is completely continuous, we can assert that $\left\{u_{n}\right\}$ is a sequentially compact set.

Since

$$
\begin{aligned}
u_{1}(t) & =T u_{0}(t) \\
& =\sum_{s=\nu-1}^{b+\nu-2} \bar{J}(t, s) \varphi_{q}\left(\frac{\lambda}{\Gamma(\beta)} \sum_{\tau=\nu-\beta-1}^{s-\beta}(s-\tau-1) \frac{\beta-1}{2} \lambda f\left(\tau^{\prime},{ }_{b+\varepsilon-1} \nabla^{-\varepsilon} u_{0}\left(\tau^{\prime}\right), u_{0}\left(\tau^{\prime}+\varepsilon\right)\right)\right) \\
& \leq \sum_{s=\nu-1}^{b+\nu-2} \bar{J}(t, s) \varphi_{q}\left(\frac{1}{\Gamma(\beta)} \lambda \sigma k^{r_{1}} a^{r_{1}+r_{2}} \sum_{\tau=\nu-\beta-1}^{s-\beta}(s-\tau-1) \frac{\beta-1}{-}\right) \\
& \leq \frac{u_{0}(t) B}{a} \varphi_{q}\left(\lambda \sigma k^{r_{1}} a^{r_{1}+r_{2}}\right) \varphi_{q}\left(\frac{(b+\beta-1)^{\underline{\beta}}}{\Gamma(\beta+1)}\right)
\end{aligned}
$$




$$
\begin{aligned}
& =\frac{u_{0}(t) N}{a} \varphi_{q}\left(\lambda \sigma k^{r_{1}} a^{r_{1}+r_{2}}\right) \\
& \leq u_{0}(t)
\end{aligned}
$$

and

$$
\begin{aligned}
& \left|{ }_{b+\varepsilon-1} \nabla^{v-\varepsilon} u_{1}(t)\right| \\
& =\varphi_{q}\left(\triangle_{\nu-\beta-1}^{-\beta} \lambda f\left(t^{\prime}{ }_{b+\varepsilon-1} \nabla^{-\varepsilon} u_{0}\left(t^{\prime}\right), u_{0}\left(t^{\prime}+\varepsilon\right)\right)\right) \\
& \leq \varphi_{q}\left(\lambda \sigma k^{r_{1}} a^{r_{1}+r_{2}}\right) \varphi_{q}\left(\frac{(b+\beta-1)^{\underline{\beta}}}{\Gamma(\beta+1)}\right) \\
& \leq \frac{a}{N} \varphi_{q}\left(\frac{(b+\beta-1)^{\underline{\beta}}}{\Gamma(\beta+1)}\right)=\frac{a}{B} \leq a,
\end{aligned}
$$

we obtain $u_{1}(t) \leq u_{0}(t),\left.\right|_{b+\varepsilon-1} \nabla^{\nu-\varepsilon} u_{1}(t)|\leq|_{b+\varepsilon-1} \nabla^{\nu-\varepsilon} u_{0}(t) \mid$ and

$$
\begin{aligned}
& u_{2}(t)=T u_{1}(t) \leq T u_{0}(t)=u_{1}(t), \\
& \left|{ }_{b+\varepsilon-1} \nabla^{v-\varepsilon} u_{2}(t)\right|=\left.\left.\right|_{b+\varepsilon-1} \nabla^{v-\varepsilon} T u_{1}(t)|\leq|_{b+\varepsilon-1} \nabla^{v-\varepsilon} T u_{0}(t)|=|\right|_{b+\varepsilon-1} \nabla^{v-\varepsilon} u_{1}(t) \mid, \\
& \quad t \in[\varepsilon, \varepsilon+b]_{\mathbb{N}_{\varepsilon}} .
\end{aligned}
$$

By induction we get

$$
u_{n+1}(t) \leq u_{n}(t),\left.\right|_{b+\varepsilon-1} \nabla^{v-\varepsilon} u_{n+1}(t)|\leq|_{b+\varepsilon-1} \nabla^{v-\varepsilon} u_{n}(t) \mid, t \in[\varepsilon, \varepsilon+b]_{\mathbb{N}_{\varepsilon}}, \quad n=0,1,2, \ldots
$$

Thus, there exists $u^{*} \in \bar{P}_{a}$ such that $u_{n} \rightarrow u^{*}$. Applying the continuity of $T$ and $u_{n+1}(t)=$ $T u_{n}(t)$, we get $T u^{*}(t)=u^{*}(t)$, which implies that $u^{*}$ is a nonnegative solution of FBVP (2.7).

On the other hand, let $w_{0}=0, t \in[\varepsilon, \varepsilon+b]_{\mathbb{N}_{\varepsilon}}$, then $w_{0} \in \bar{P}_{a}$. Let $w_{1}=T w_{0}$, then $w_{1} \in \bar{P}_{a}$. Denote

$$
w_{n+1}=T w_{n}=T^{n+1} w_{0}, \quad n=0,1,2, \ldots
$$

It follows from $T \bar{P}_{a} \subset \bar{P}_{a}$ that $w_{n} \in \bar{P}_{a}, n=0,1,2, \ldots$ Since $T$ is completely continuous, we can assert that $\left\{w_{n}\right\}$ is a sequentially compact set.

Since $w_{1}=T w_{0} \in \bar{P}_{a}$, we have

$$
w_{1}(t)=\left(T w_{0}\right)(t) \geq w_{0}, \quad t \in[\varepsilon, \varepsilon+b]_{\mathbb{N}_{\varepsilon}} .
$$

So

$$
\begin{aligned}
& w_{2}(t)=\left(T w_{1}\right)(t) \geq w_{1}, \quad t \in[\varepsilon, \varepsilon+b]_{\mathbb{N}_{\varepsilon}}, \\
& \left|{ }_{b+\varepsilon-1} \nabla^{\nu-\varepsilon} w_{2}(t)\right|=\left|{ }_{b+\varepsilon-1} \nabla^{\nu-\varepsilon}\left(T w_{1}\right)(t)\right| \geq\left|{ }_{b+\varepsilon-1} \nabla^{\nu-\varepsilon} w_{1}(t)\right|, \quad t \in[v, v+b]_{\mathbb{N}_{v}} .
\end{aligned}
$$

By induction we get

$$
w_{n+1}(t) \geq w_{n}(t), \quad\left|{ }_{b+\varepsilon-1} \nabla^{\nu-\varepsilon} w_{n+1}(t)\right| \geq\left.\right|_{b+\varepsilon-1} \nabla^{\nu-\varepsilon} w_{n}(t) \mid, \quad n=0,1,2, \ldots
$$


Hence, there exists $w^{*} \in \bar{P}_{a}$ such that $w_{n} \rightarrow w^{*}$. Applying the continuity of $T$ and $w_{n+1}(t)=$ $T w_{n}(t)$, we obtain $T w^{*}(t)=w^{*}(t)$, which implies that $w^{*}$ is a nonnegative solution of FBVP (2.7).

Thus FBVP (2.7) has two positive solutions $u^{*}, w^{*}$ such that $0 \leq\left\|u^{*}\right\| \leq a, 0 \leq\left\|w^{*}\right\| \leq a$, and from the above proof, we know that the iterative sequences hold.

In order to illustrate the main result, we give the following example.

Example 4.1 Consider the following FBVP:

$$
\begin{aligned}
& \triangle_{-\frac{1}{2}}^{\frac{1}{2}}\left(\varphi_{3}\left(\frac{4}{3} \nabla^{\frac{7}{6}} y(t)\right)\right)=-\left[e^{t}\left(\left({ }_{\frac{4}{3}} \nabla^{-\frac{1}{3}} y(t)\right)^{\frac{3}{5}}+y^{\frac{1}{3}}\left(t+\frac{1}{3}\right)\right)+1\right] \\
& y\left(\frac{3}{7}\right)=0, \quad{ }_{\frac{4}{3}} \nabla^{\frac{7}{6}} y\left(-\frac{1}{2}\right)=0, \quad{ }_{\frac{4}{3}} \nabla^{-\frac{1}{3}} y(-1)=\sum_{t=0}^{1}{ }_{\frac{4}{3}} \nabla^{-\frac{1}{3}} y(t) A(t),
\end{aligned}
$$

where $p=3, v=\frac{3}{2}, \beta=\frac{1}{2}, \varepsilon=\frac{1}{3}, \lambda=1, b=2$,

$$
A(t)= \begin{cases}0, & t=0 \\ 1, & t=1 \\ 2, & t=2 .\end{cases}
$$

Let

$$
f(t, u, s)=e^{t}\left(u^{\frac{3}{5}}+s^{\frac{1}{3}}\right)+1, \quad r_{1}=\frac{3}{5}, \quad r_{2}=\frac{1}{3},
$$

then for any $\lambda \in(0,1)$ and $u, s \in[0,+\infty), t \in[0,2]_{\mathbb{N}_{0}}$, we have

$$
\begin{aligned}
& f(t, \lambda u, s)=e^{t}\left[(\lambda u)^{\frac{3}{5}}+s^{\frac{1}{3}}\right]+1 \geq \lambda^{\frac{3}{5}}\left[e^{t}\left(u^{\frac{3}{5}}+s^{\frac{1}{3}}\right)+1\right] \geq \lambda^{\frac{3}{5}} f(t, u, s), \\
& f(t, u, \lambda s)=e^{t}\left[u^{\frac{3}{5}}+(\lambda s)^{\frac{1}{3}}\right]+1 \geq \lambda^{\frac{1}{3}}\left[e^{t}\left(u^{\frac{3}{5}}+s^{\frac{1}{3}}\right)+1\right] \geq \lambda^{\frac{1}{3}} f(t, u, s),
\end{aligned}
$$

which implies that $\left(H_{6}\right)$ holds.

On the other hand, it is clear that $\left(H_{1}\right),\left(H_{2}\right)$ and $\left(H_{3}\right)$ are satisfied, and

$$
\sigma=\max _{t \in[0,2]]_{\mathbb{N}}} f(t, 1,1)=\max _{t \in[0,2]_{\mathbb{N}}}\left(2 e^{t}+1\right)=2 e^{2}+1
$$

Next we compute $k, C, B$ and $N$. We have

$$
\begin{aligned}
& C=\frac{8}{15}, \quad k=\frac{4}{3} \\
& \sum_{s=\frac{1}{2}}^{\frac{3}{2}} \bar{J}\left(\frac{1}{3}, s\right)=\frac{4}{9}\left(\frac{\Gamma\left(\frac{1}{6}\right)}{\Gamma\left(\frac{2}{3}\right) \Gamma\left(\frac{1}{2}\right)}+\frac{8}{\Gamma\left(\frac{1}{2}\right)}\right), \quad \sum_{s=\frac{1}{2}}^{\frac{3}{2}} \bar{J}\left(\frac{4}{3}, s\right)=\frac{4}{9} \frac{\Gamma\left(\frac{1}{6}\right)}{\Gamma\left(\frac{2}{3}\right) \Gamma\left(\frac{1}{2}\right)}+\frac{64}{21 \Gamma\left(\frac{1}{2}\right)}, \\
& \sum_{s=\frac{1}{2}}^{\frac{3}{2}} \bar{J}\left(\frac{7}{3}, s\right)=0
\end{aligned}
$$


so

$$
\begin{aligned}
& B=\max \left\{\max _{t \in[\varepsilon, \varepsilon+b]_{\mathbb{N}_{\varepsilon}}} \sum_{s=\nu-1}^{b+v-2} \bar{J}(t, s), 1\right\}=\frac{4}{9}\left(\frac{\Gamma\left(\frac{1}{6}\right)}{\Gamma\left(\frac{2}{3}\right) \Gamma\left(\frac{1}{2}\right)}+\frac{8}{\Gamma\left(\frac{1}{2}\right)}\right), \\
& N=B \varphi_{q}\left(\frac{3}{2}\right), \quad \varphi_{p}(N)=\frac{3}{2} \varphi_{p}(B)=\frac{8}{27}\left(\frac{\Gamma\left(\frac{1}{6}\right)}{\Gamma\left(\frac{2}{3}\right) \Gamma\left(\frac{1}{2}\right)}+\frac{8}{\Gamma\left(\frac{1}{2}\right)}\right)^{2} .
\end{aligned}
$$

Take $a=\left[\frac{1}{2}\left(\frac{\Gamma\left(\frac{1}{6}\right)}{\Gamma\left(\frac{2}{3}\right) \Gamma\left(\frac{1}{2}\right)}+\frac{8}{\Gamma\left(\frac{1}{2}\right)}\right)^{2}\left(2 e^{2}+1\right)\right]^{\frac{15}{16}}$, then

$$
\frac{\varphi_{p}(a)}{\sigma a^{r_{1}+r_{2}} k^{r_{1}}} \approx 0.42\left(\frac{\Gamma\left(\frac{1}{6}\right)}{\Gamma\left(\frac{2}{3}\right) \Gamma\left(\frac{1}{2}\right)}+\frac{8}{\Gamma\left(\frac{1}{2}\right)}\right)^{2}>\frac{8}{27}\left(\frac{\Gamma\left(\frac{1}{6}\right)}{\Gamma\left(\frac{2}{3}\right) \Gamma\left(\frac{1}{2}\right)}+\frac{8}{\Gamma\left(\frac{1}{2}\right)}\right)^{2}=\varphi_{p}(N)
$$

which implies that $\left(H_{8}\right)$ holds. For $a=\left[\frac{1}{2}\left(\frac{\Gamma\left(\frac{1}{6}\right)}{\Gamma\left(\frac{2}{3}\right) \Gamma\left(\frac{1}{2}\right)}+\frac{8}{\Gamma\left(\frac{1}{2}\right)}\right)^{2}\left(2 e^{2}+1\right)\right]^{\frac{15}{16}}$, it is clear that $\left(H_{7}\right)$ holds. So by Theorem 4.1, FBVP (4.6) and (4.7) has two solutions $u^{*}$ and $w^{*}$ such that

$$
\begin{aligned}
& 0<u^{*}<\left[\frac{1}{2}\left(\frac{\Gamma\left(\frac{1}{6}\right)}{\Gamma\left(\frac{2}{3}\right) \Gamma\left(\frac{1}{2}\right)}+\frac{8}{\Gamma\left(\frac{1}{2}\right)}\right)^{2}\left(2 e^{2}+1\right)\right]^{\frac{15}{16}}, \\
& 0<\left|\frac{4}{3} \nabla^{-\frac{1}{3}} u^{*}\right|<\left[\frac{1}{2}\left(\frac{\Gamma\left(\frac{1}{6}\right)}{\Gamma\left(\frac{2}{3}\right) \Gamma\left(\frac{1}{2}\right)}+\frac{8}{\Gamma\left(\frac{1}{2}\right)}\right)^{2}\left(2 e^{2}+1\right)\right]^{\frac{15}{16}}, \\
& \lim _{n \rightarrow \infty} u_{n}=\lim _{n \rightarrow \infty} T^{n} u_{0}=u^{*}, \quad \lim _{n \rightarrow \infty}\left(\frac{4}{3} \nabla^{-\frac{1}{3}} u_{n}\right)=\lim _{n \rightarrow \infty} \frac{4}{3} \nabla^{-\frac{1}{3}} T^{n} u_{0}=\frac{4}{3} \nabla^{-\frac{1}{3}} u^{*},
\end{aligned}
$$

where

$$
\begin{aligned}
u_{0}(t)= & \frac{9}{4}\left(\frac{2 e^{2}+1}{2}\right)^{\frac{15}{16}}\left[\frac{\Gamma\left(\frac{1}{6}\right)}{\Gamma\left(\frac{2}{3}\right) \Gamma\left(\frac{1}{2}\right)}+\frac{8}{\Gamma\left(\frac{1}{2}\right)}\right]^{\frac{7}{8}} \\
& \times \begin{cases}\frac{8\left(\frac{3}{2}-t\right)^{\frac{1}{2}}}{3 \Gamma\left(\frac{1}{2}\right)}-\frac{2\left(\frac{1}{2}-t\right) \frac{1}{2}}{\Gamma\left(\frac{1}{2}\right)}+\frac{128\left(\frac{3}{2}-t\right) \frac{1}{6}}{7 \Gamma\left(\frac{1}{2}\right) \Gamma\left(\frac{1}{6}\right)}, & t \in[0,1), \\
\frac{8\left(\frac{3}{2}-t\right) \frac{1}{2}}{3 \Gamma\left(\frac{1}{2}\right)}+\frac{128\left(\frac{3}{2}-t\right) \frac{1}{6}}{7 \Gamma\left(\frac{1}{2}\right) \Gamma\left(\frac{1}{6}\right)}, & t \in[1,2],\end{cases}
\end{aligned}
$$

and

$$
\begin{aligned}
& 0<w^{*}<\left[\frac{1}{2}\left(\frac{\Gamma\left(\frac{1}{6}\right)}{\Gamma\left(\frac{2}{3}\right) \Gamma\left(\frac{1}{2}\right)}+\frac{8}{\Gamma\left(\frac{1}{2}\right)}\right)^{2}\left(2 e^{2}+1\right)\right]^{\frac{15}{16}}, \\
& 0<\left|\frac{4}{3} \nabla^{-\frac{1}{3}} w^{*}\right|<\left[\frac{1}{2}\left(\frac{\Gamma\left(\frac{1}{6}\right)}{\Gamma\left(\frac{2}{3}\right) \Gamma\left(\frac{1}{2}\right)}+\frac{8}{\Gamma\left(\frac{1}{2}\right)}\right)^{2}\left(2 e^{2}+1\right)\right]^{\frac{15}{16}}, \\
& \lim _{n \rightarrow \infty} w_{n}=\lim _{n \rightarrow \infty} T^{n} w_{0}=w^{*}, \quad \lim _{n \rightarrow \infty}\left(\frac{4}{3} \nabla^{-\frac{1}{3}} w_{n}\right)=\lim _{n \rightarrow \infty} \frac{4}{3} \nabla^{-\frac{1}{3}} T^{n} w_{0}=\frac{4}{3} \nabla^{-\frac{1}{3}} w^{*},
\end{aligned}
$$

where $w_{0}(t)=0, t \in[0,2]_{\mathbb{N}_{0}}$.

\section{Competing interests}

The authors declare that they have no competing interests.

\section{Authors' contributions}

$\mathrm{HL}$ and $\mathrm{CH}$ worked together in the derivation of the mathematical results. All authors read and approved the final manuscript. 


\section{Acknowledgements}

The authors would like to thank the referees for invaluable comments and insightful suggestions. Project supported by the National Natural Science Foundation of China (Chengmin Hou - 11161049; Yuanfeng Jin -11361066).

\section{Publisher's Note}

Springer Nature remains neutral with regard to jurisdictional claims in published maps and institutional affiliations.

Received: 6 December 2016 Accepted: 5 March 2017 Published online: 21 April 2017

\section{References}

1. Mastorakis, NE, Fathabadi, H: On the solution of $p$-Laplacian for non-Newtonian fluid flow. WSEAS Trans. Math. 8(6), 238-245 (2009)

2. Chhetri, M, Oruganti, S, Shivaji, R: Positive solutions for classes of $p$-Laplacian equations. Differ. Integral Equ. 17(11-12), 1255-1261 (2004)

3. Oruganti, S, Shi, J, Shivaji, R: Logistic equation with the p-Laplacian and constant yield harvesting. Abstr. Appl. Anal. 2004(9), 723 (2004)

4. Liu, C: Weak solutions for a viscous p-Laplacian equation. Electron. J. Differ. Equ. 2003(1), 1 (2003)

5. Evans, LC, Gangbo, W: Differential equations methods for the Monge-Kantorovich mass transfer problem. Mem. Am. Math. Soc. 137, 653 (1999)

6. Nieto, JJ, Pimentel, J: Positive solutions of a fractional thermostat model. Bound. Value Probl. 2013(1), 1 (2013)

7. Ding, $\mathrm{XL}$, Jiang, $Y \mathrm{~L}$ : Waveform relaxation methods for fractional functional differential equations. Fract. Calc. Appl. Anal. 16(3), 573-594 (2013)

8. Miller, KS, Ross, B: An Introduction to the Fractional Calculus and Fractional Differential Equations. Wiley, New York (1993)

9. Nisar, KS, Baleanu, D, Qurashi, MMA: Fractional calculus and application of generalized Struve function. SpringerPlus 5(1), 1-13 (2016)

10. Oldham, KB, Spanier, J: The Fractional Calculus: Theory and Applications of Differentiation and Integration to Arbitrary Order. Dover, New York (2002)

11. Virginia, K: The special functions of fractional calculus as generalized fractional calculus operators of some basic functions. Comput. Math. Appl. 59(3), 1128-1141 (2010)

12. Guy, J: On the derivative chain-rules in fractional calculus via fractional difference and their application to systems modelling. Open Phys. 11(6), 617-633 (2016)

13. Pilipović, S: Fractional calculus with applications in mechanics: vibrations and diffusion processes. Drug Dev. Ind. Pharm. (2014)

14. Atici, FM, Eloe, PW: A transform method in discrete fractional calculus. Int. J. Difference Equ. 2, 165-176 (2007)

15. Goodrich, SC: Solutions to a discrete right-focal fractional boundary value problem. Int. J. Difference Equ. 5(2), 195-216 (2010)

16. Goodrich, C, Peterson, AC: Discrete Fractional Calculus Springer, Berlin (2015). ISBN:978-3-319-25562-0

17. Ibrahim, RW, Jalab, HA: Existence of a class of fractional difference equations with two point boundary value problem. Adv. Differ. Equ. 2015, 269 (2015)

18. Xie, Z, Hou, C: Properties of right fractional sum and right fractional difference operators and application. Adv. Differ Equ. 2015, 288 (2015)

19. Goodrich, CS: Systems of discrete fractional boundary value problems with nonlinearities satisfying no growth conditions. J. Differ. Equ. Appl. 21(5), 437-453 (2015)

20. Lin, X, Zhao, Z, Guan, Y: Iterative technology in a singular fractional boundary value problem with $q$-difference. Appl. Math. (Irvine) 7(1), 91-97 (2016)

21. Malinowska, AB, Torres, DFM: The delta-nabla calculus of variations. Fasc. Math. 44(44), 75-83 (2009)

22. Natália, M, Torres, DFM: Calculus of variations on time scales with nabla derivatives. Nonlinear Anal. 71(12), e763-e773 (2008)

23. Lv, W: Existence of solutions for discrete fractional boundary value problems with a $p$-Laplacian operator. Adv. Differ Equ. 2012(1), 163 (2012)

24. Xie, Z, Jin, Y, Hou, C: Multiple solutions for a fractional difference boundary value problem via variational approach. Abstr. Appl. Anal. 2012, 137 (2012)

\section{Submit your manuscript to a SpringerOpen ${ }^{\ominus}$ journal and benefit from:}

- Convenient online submission

- Rigorous peer review

- Immediate publication on acceptance

- Open access: articles freely available online

- High visibility within the field

- Retaining the copyright to your article 